\title{
Optimization of Steel Production Scheduling with Complex Time- Sensitive Electricity Cost
}

Hubert Hadera ${ }^{\mathrm{a}, \mathrm{b}}$, Iiro Harjunkoski ${ }^{\mathrm{a} *}$, Guido Sand ${ }^{\mathrm{a}}$, Ignacio E. Grossmann ${ }^{\mathrm{c}}$, Sebastian Engell ${ }^{\mathrm{b}}$

${ }^{a}$ ABB Corporate Research, Wallstadter Str. 59, 68526 Ladenburg, Germany

${ }^{b}$ Technische Universität Dortmund, Emil-Figge Str. 70, 44221 Dortmund, Germany

${ }^{c}$ Carnegie Mellon University, 5000 Forbes Ave., Pittsburgh, PA 15213, USA

*Iiro.Harjunkoski@de.abb.com

\begin{abstract}
Energy-intensive industries can take advantage of process flexibility to reduce operating costs by optimal scheduling of production tasks. In this study we develop a MILP formulation to extend a continuous-time model with energy-awareness to optimize the daily production schedules and the electricity purchase including the load commitment problem. The sources of electricity that are considered are purchase on volatile day-ahead markets, time-of-use (TOU) contracts and base load contracts, as well as onsite generation. The possibility to sell electricity back to the grid is also included. The model is applied to the melt shop section of a stainless steel plant. Due to the large-scale nature of the combinatorial problem which prevents the solution of a monolithic model to nearoptimality, we propose a bi-level heuristic algorithm to tackle instances of industrial size. Case study scenarios show that the potential impact of high prices in the day-ahead markets of electricity can be mitigated by jointly optimizing the production schedule and the associated net electricity consumption cost.
\end{abstract}

Keywords: scheduling, steel plant, energy optimization, demand-side management, continuous-time models. 


\section{Introduction}

In many countries, renewable energy sources contribute a significant share of the overall electric power consumption and due to the volatility of their availability and their privileged role on the market, this may cause high fluctuations of the energy cost for the final user. On the grid level, the demand should always match the supply, otherwise the grid infrastructure is stressed, possibly causing expensive failures. Therefore it is of interest to the supply side of the grid to achieve flexibility of the demand, which traditionally was assumed to be inelastic in the short-term. This is largely because the consumers of electricity were not getting incentive signals which could trigger changes of the consumption pattern when shortages or oversupply occur. In recent times however, smart grid technologies and the liberalization of the energy markets provided new ways of communicating such signals, both for dispatchable loads (the user is given direct signals to change the consumption) and for non-dispatchable loads (the user decides whether to change the consumption) (NERC 2007). The latter signals are considered in this work in the form of financial incentives and different pricing contracts. These fall within the Demand-Side Management technology, which aims at supporting an active shaping of the patterns of energy use. In particular, industrial Demand-Side Response (iDSR) involves activities defined as a temporary change in electricity consumption in response to market or supply conditions. In non-dispatchable iDSR a consumer, e.g. a steel plant is allowed to decide whether it wants to react to a changing situation within the grid, potentially gaining financial benefits, or to stick to the production plan. This implies the need of proper day-to-day scheduling and planning of plant operations, and for making use of incentive and price based schemes, such as for example intra-day or day-ahead spot market pricing since changes in the prices of energy might significantly affect the profitability as shown for a stainless-steel production plant in Hadera et al. (2014). If it is assumed that the goal of the plant managers is to deliver the same amount of final products over a certain time horizon, the production schedule can be modified in favor of a lower cost of energy procurement only when the process-specific constraints are always satisfied, and when at the same time the plant faces a certain under-utilization of its production capacity. As shown in Figure 1, the capacity utilization of the US-based energy-intensive primary metal sector went down by nearly $20 \%$ in recent years compared to 1990's (BGFRS 2013).

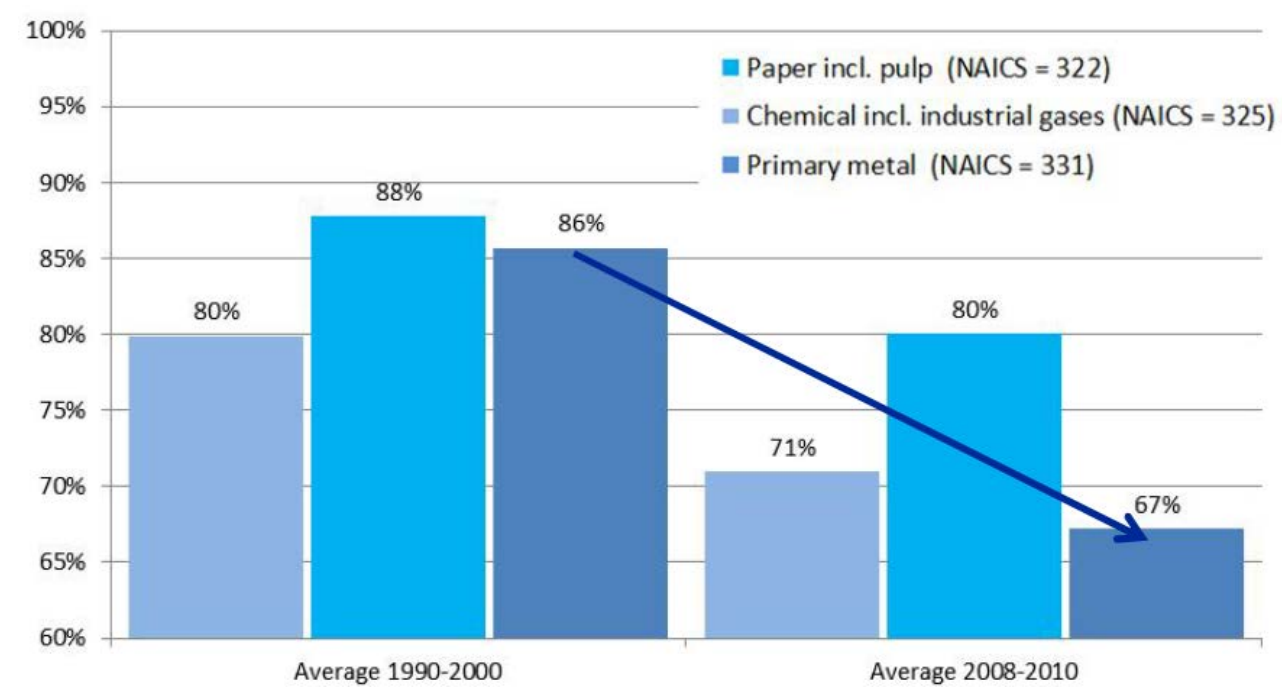

Figure 1 Capacity utilization of US Energy-Intensive Industries (ABB study based on BGFRS 2013)

This creates a potential to optimally shift the production to times when the consumption of electricity is cheaper. This is especially valid for energy-intensive process industries where the raw material and 
energy cost can account for up to $90 \%$ of the total production cost. Demand-response technology on the production scheduling and planning level has the advantage of a potentially low investment cost for the final user, since very often it does not require the purchase of new equipment. Other selected positive outcomes of a more flexible Demand-side Response that are reported in the literature are (NECR 2007, CRA 2005, Todd et al. 2009):

- Plant level: direct cost savings on the electricity bill;

- Grid level: increase of reliability, e.g. reduction of outages;

- Grid level: reduction of expensive peak load hours in the short-term;

- Environment: potential emission savings by reducing the grid's peak generation (only for regions with fossil fired peak generation plants);

- Environment: potential reduction of emissions by enabling the installation of larger renewable generation capacities;

- Market: market-wide wholesale electricity price reduction in the long-term;

- Market: market performance benefits, e.g. mitigating the suppliers' ability to raise prices significantly above production costs.

Except for the direct energy bill cost savings at the plant site, quantification of the above benefits is difficult and strongly depends on assumptions. However, industrial and academic studies conclude that the potential exists (DOE 2006, NERC 2007, DENA 2011). When investigating DSR of industrial production, it is important to consider the technical potential of Demand-side Response shaping capabilities and not only the total consumption of the process, as pointed out by Paulus and Borggrefe (2011). An ideal industrial plants should have large consumers of electric power that operate in a preplanned fashion and a degree of process flexibility. Both hold true for the steel plant considered in this paper.

While the iDSR technology is recognized as beneficial for both the power supplier side and for the energy-intensive industry, it should be noted that it cannot compensate long-term deficits or surplus of electricity generation in regional grids.

\subsection{Scope and methodology}

The goal of this work is to find an optimal production schedule of a part of a steel making process that is operated in batch mode which minimizes a weighted combination of the electricity bill and the lead times of product delivery, while satisfying complex production constraints. In the case considered here, a continuous-time based general precedence scheduling approach has already been developed for the plant which is extended here to include awareness of the cost of electric energy. The goal is to enable an energy-intensive process plant to realize its demand-side response potential at the production scheduling level, finding a compromise between production delays and the cost of electricity. The main contributions of this work concerns the development of the following items:

- A generic strategy for energy-aware scheduling, accounting for time-depending cost of energy in general precedence continuous-time scheduling models;

- Extension of generic multiple purchase contracts optimization to energy-aware scheduling;

- A bi-level heuristic for obtaining good solutions in reasonable times for industrial scale combined production scheduling and energy cost minimization problems.

The benefit of using a continuous-time formulation is the exact timing of the production tasks within the scheduling horizon. This is in contrast to discrete-time approaches, which discretize the time horizon into discrete time intervals. From industrial practice we consider 5-minute discretization steps as the desired level of time granularity. Such small time window creates very large discrete-time 
models leading to computational limitations. Other studies showed that a 15-minute discretization (Castro et al. 2013) can still be efficient for solving 24h scheduling horizon with a Resource-Task Network (RTN) based monolithic model approach. However, compared to the discrete-time formulation, continuous-time models also have some drawbacks. Due to the structure of the pricing contracts, it is much easier to account for the cost of the consumption of electricity in discrete-time scheduling models. Extending it to continuous-time formulations is not straightforward since the use of electricity has to be accounted for in fixed time intervals in which related to the resource prices are constant.

In this paper we consider purchase optimization of multiple sources of electricity, including the possibility to sell the electricity back to the grid. Also, the challenge of responding to a committed load curve with penalties incurred for both under- and over-consumption is addressed. The combination of these two features has not received much attention in the process scheduling literature yet. For the given multi-stage steel plant with parallel machines at each stage, the resulting monolithic formulation of the problem is computationally intractable when the scheduling decisions (assignment and sequence binaries) are degrees of freedom for the optimization. To overcome the computational limitations we introduce a simple bi-level heuristic approach. The problem is modeled using mathematical programing with Mixed-Integer Linear Programing (MILP) and implemented in the GAMS modeling environment using the CPLEX solver.

In the remaining sections, the background and previous work in the area and the industrial problem are described first. Then the section of the process that is considered and the corresponding energy purchase situation are explained (Section 3). Based on the continuous-time approach for batch processes, the scheduling problem is formulated so that all production constraints of the use case are satisfied (Section 4.2). In Section 4.3, we introduce a strategy of embedding energy-awareness into the continuous-time general precedence formulation. We test the resulting monolithic models (Section 5) and show that they are not able to cope with the size of the real-world problem. Therefore, in Section 6 , we describe a bi-level heuristic for the solution of the application problem. Numerical experiments and results are discussed in Section 6.5, followed by conclusions and recommendations for further work (Section 7).

\section{Literature review}

The field of scheduling and planning has grown rapidly in the last decades. A large number of studies have emerged using both time representation approaches: discrete and continuous. For a general overview concerning the scheduling problems we refer the reader to review papers, such as for example Floudas and Lin (2004), Méndez et al. (2006), Maravelias (2012) and Harjunkoski et al. (2014). The latter focuses especially on the industrial aspects of the scheduling methods.

Scheduling of steel plants has been studied quite extensively as well, as it is recognized one of the most difficult industrial scheduling problems. For handling complex process constraints and optimizing traditional objective functions such as makespan or earliest task completion time an efficient multi-step decomposition approach for the industrial-size scheduling of the melt shop area of a stainless steel plant has been reported by Harjunkoski and Grossmann (2001) based on MILP and LP models. Tang et al. (2001) gives an overview of planning and scheduling systems for integrated steel plants, including Artificial Intelligence, Expert Systems, intelligent search and Constraint Programing methods. In Li et al. (2012) the focus was on the last continuous-casting stage where particular operational features have to be addressed and a rolling horizon was used. 
In recent years scheduling under energy constraints has gained increasing attention. It has been also recognized as one of the challenges for industrial implementation of advanced scheduling solutions (Harjunkoski et al. 2014). Optimizing operations with regard to the response to a deterministic single time-varying price of electricity can be found in the literature, including also the stochastic nature of the prices, such as in Li et al. (2003) or in Ierapetritou et al (2002).

The Demand-side Response has to deal with different time scales. A fast response is required in some iDSR schemes, for example in network ancillary services. Here control techniques rather than scheduling might be better suited. As investigated by Vujanic et al. (2012), robust optimization might help creating flexible schedules to support the ancillary services of cement plants. Since energy availability and prices can be treated like any other resource in the scheduling models, many of the formulations in the literature use a discrete-time approach. Zhang and Tang (2010) introduce a discrete-time scheduling formulation using a Lagrangian relaxation algorithm based on the subgradient method. The model includes constraints concerning power availability and minimization of the energy cost. Similarly, in a study by Ashok (2006) a discrete-time formulation is used to schedule a mini steel plant where the operating cost is optimized. The operating cost includes the price of power consumption under different tariffs, charges for registered maximum demand and additional operating cost due to the shifting of loads.

In recent years, models based on the RTN representation have gained attention as an efficient way to deal with resource consumption. Castro et al. (2009) proposed a new strategy for handling variable electricity cost in continuous plants using a continuous-time formulation. Comparison of both continuous- and discrete-time RTN representations showed that the latter's computational performance is better for handling industrial-size instances. The work has been extended by an efficient rolling horizon algorithm in Castro et al. (2011) using an aggregate model, where time intervals of the same resource cost are aggregated into one interval. A steel plant scheduling problem similar to the one studied in this paper, but with response to a single price curve, has been successfully reported by Castro et al. (2013) for a time granularity of 15 minutes intervals.

Nolde and Morari (2010) proposed a strategy for the modeling of electricity consumption with timedependent prices in continuous-time models based on precedence variables. It was applied to a stainless-steel process with parallel Electric-Arc Furnaces. The formulation uses six different binary variables to capture the relation of a production task to its placement within a grid of uniform time intervals. For these intervals electricity consumption is individually accounted for, which makes it possible to track the process load and to optimize the deviation from a pre-agreed consumption curve. Haït and Artigues (2011a) proposed an improvement to Nolde and Morari's approach replacing the set of six binary variables by binaries indicating whether or not an event takes place before or during a time interval. For the same steel case problem, the resulting continuous-time MILP model introduced fewer number of constraints and binary variables. As a follow up study on scheduling of a foundry, Haït and Artigues (2011b) proposed a hybrid heuristic combining Constraint Programing (CP) for solving the assignment and sequencing problem with an MILP model for solving the remaining energy-cost scheduling problem. In addition, the detailed scheduling of the Electric Arc Furnace stage and human operator availability were taken into consideration. Castro et al. (2014) applied the concept of the six cases of task-time interval relations as in Nolde and Morari (2010) to optimize the maintenance of a gas-fired power plant. Using Generalized Disjunctive Programming, Castro and coworkers found a tighter formulation for the accounting of electricity consumption. The continuoustime strategy was applied to find a schedule under constraints of operator availability and cost, maximizing profits from electricity sales under time-sensitive demand and pricing. A steel plant was also considered in a study by Boukas et al. (1990), using a hierarchical approach with separation of 
operation and secondary resource scheduling in two steps. Constraints were subject to a global limitation of the power delivered to the furnaces.

Apart from the steel industry, demand-side response strategies have been investigated for other energy-intensive processes. Mitra et al. (2012) proposed a discrete-time formulation for process plants with an emphasis on switching the operating modes of the plant units. Responding to a single timesensitive price curve of electricity the model was successfully applied to air separation and cement production processes. The same solution strategy was also applied in the context of optimal scheduling of an industrial Combined Heat and Power (CHP) plant (Mitra et al. (2013). Underutilization of the CHP plant and its response to time-sensitive electricity prices were investigated.

\section{Problem description}

The industrial problem that is addressed in this work concerns the optimal scheduling of a part of the stainless-steel production process. The production starts with the scrap melting phase in an Electric Arc Furnace (EAF) to form a so-called heat which is the object of scheduling. The process of smelting is carried out by passing large amounts of electricity through electrodes in order to form hightemperature electric arc (up to $3500^{\circ} \mathrm{C}$ ) that is capable of melting scrap metal. After a full heat is formed in the EAF, the heat is transported to the next stage, the Argon Oxygen Decarburization (AOD), where the carbon content of the molten steel is reduced by injecting an argon-oxygen gas mixture. In order to ensure specific parameters of the molten steel for the final stage of casting, a heat goes through the Ladle Furnace (LF) stage to adjust the chemistry and temperature to their specified values. Finally, the heat is casted in the Continuous-casting (CC) stage, where specific rules about the sequences of heats apply. The process is shown in Figure 2.

There are several production constraints that have to be satisfied by the scheduling model formulation. We consider two parallel, non-identical machines at each stage. For all stages, except of the CC, processing of a subsequent heat can be carried out only after an equipment specific setup has been performed. Between subsequent stages, a heat is transported with some minimum time requirement which differs depending on the two units considered. The time spent by a heat waiting between two subsequent stages is restricted by a maximum allowed hold-up time in order to avoid a too-large drop of the temperature of the molten steel. Heats of the same heat group are casted subsequently on the CC without waiting times.

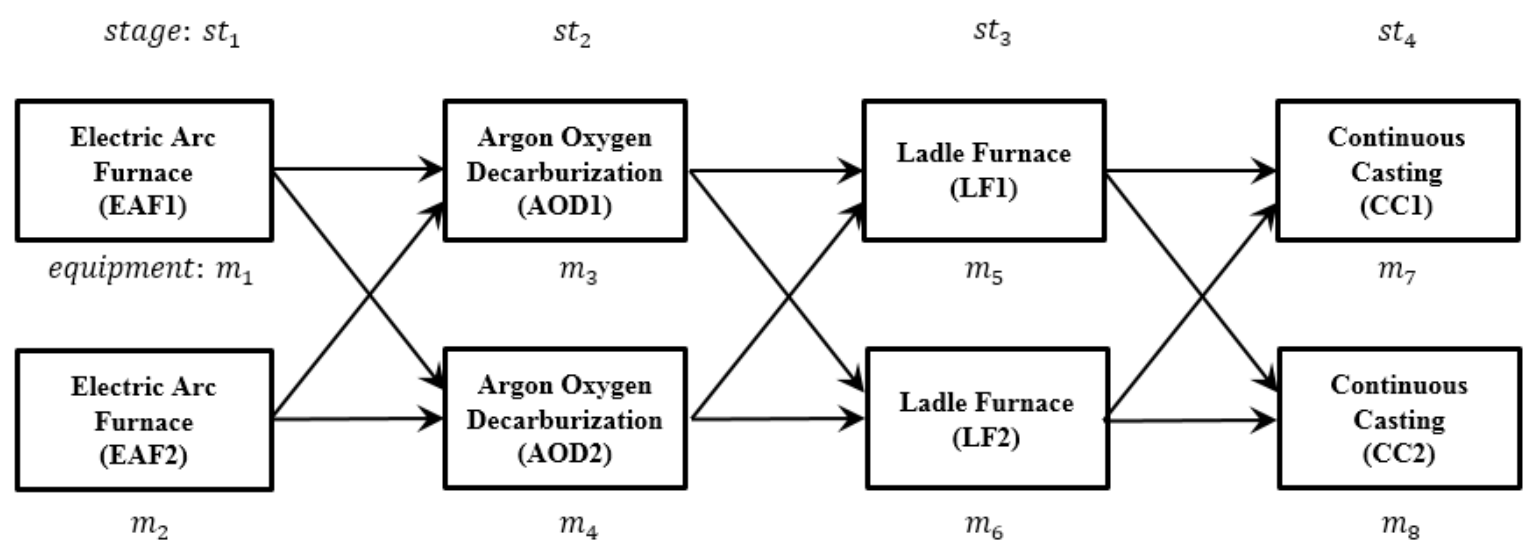

Figure 2 Stainless-steel production process (melt shop section) 


\subsection{Electricity demand considerations}

The above mentioned production process consumes large amounts of electricity, in the considered case up to $192 \mathrm{MW}$. The energy demand for this process must always be met, i.e. the plant is assumed to purchase at least the amount of electricity needed to satisfy the load curve that results from the production schedule. We consider demand-side response strategies which preserve the total production output over some given time horizon, in the computational studies over one day. The challenge addressed in this work is to determine simultaneously an optimal purchase and sales policy for the electricity, with complex time- and load-sensitive purchasing options as shown in Figure 3 and a production schedule that defines the demand of electricity.
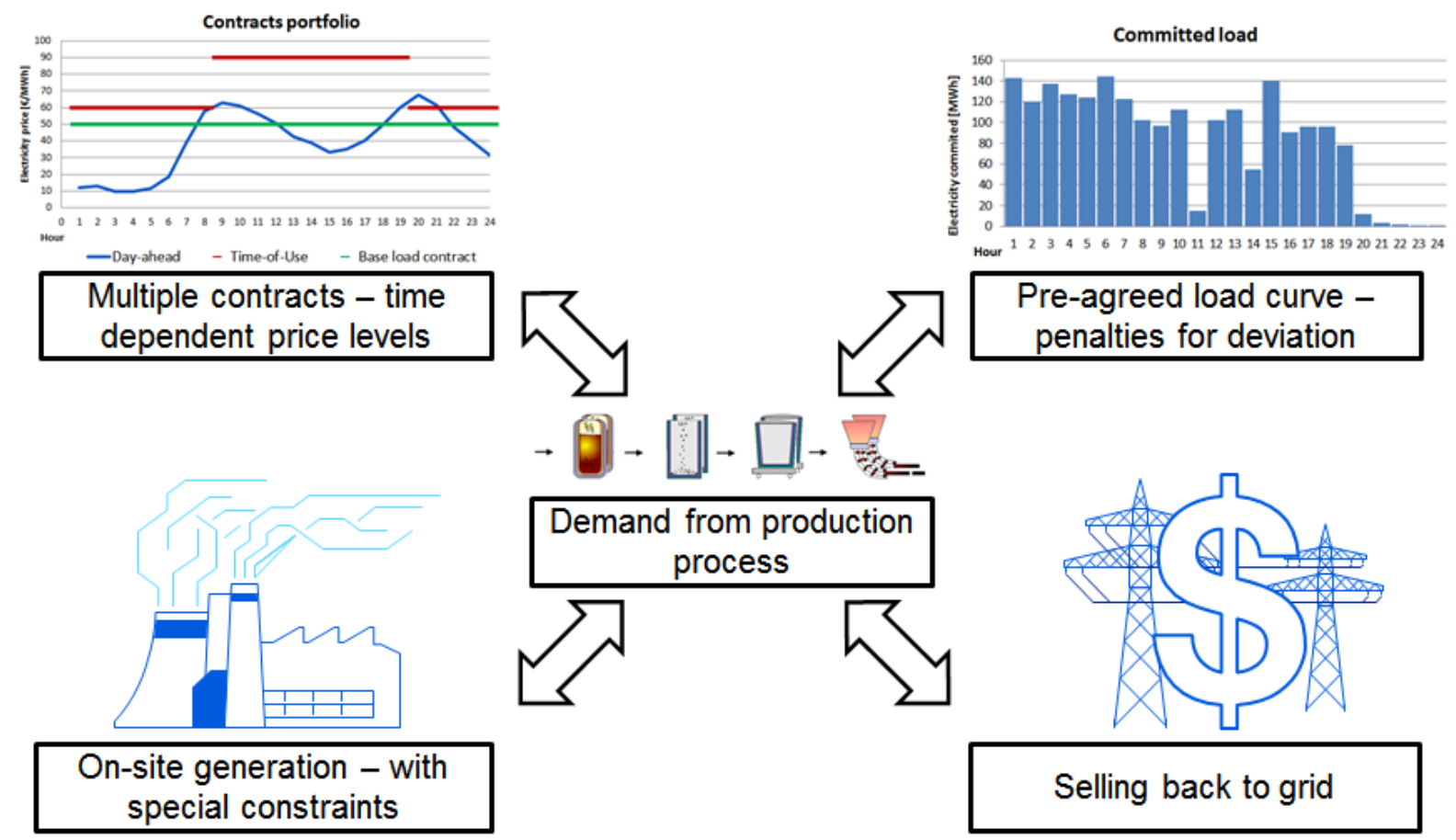

Figure 3 Electricity bill structure

For the industrial case study the purchasing contracts include:

- long-term contract (base contract or base load) - constant price, constant amount of electricity delivered over time;

- $\quad$ short-term contract (Time-of-Use or TOU) - two price levels (on- and off-peak);

- $\quad$ spot market (day-ahead) - hourly-varying prices, known 24 hours ahead;

- onsite generation- constant price with additional start-up costs.

The long-term contract is agreed with a provider usually for a period of 3-12 months. Over this time a certain fixed amount of electricity is available for the production plant at all times. The agreed amount must be purchased by the plant. Therefore, in a situation where there is no load consumption planned at some time interval, the surplus of electricity must be sold back to the grid. Establishing a long-term contract is usually considered profitable for the plant since the provider is able to offer a lower price for such a constant delivery over a long period.

The short-term contract (TOU) usually covers up to 3 months. Therefore, the price offered by the supplier is normally higher compared to the long-term contract. Here, we assume that the contract has two different price levels corresponding to on- and off-peak times. The off-peak price is lower than the 
on-peak price which applies during the daylight period. Another contract considered in the case study is the day-ahead spot market. Here, the price follows regional fluctuations of electricity availability; therefore, it varies on an hourly basis.

Apart from purchase contracts, the plant may have the possibility to produce electricity internally, which is subject to additional constraints. A start-up cost needs to be accounted for in the total cost of onsite generation for each time the onsite power generation is started up. Also, minimum runtime and downtime restrictions apply to avoid frequent start-ups and shut-downs of the power plant which lead to an accelerated deterioration of the plant.

The total electricity bill can be reduced by selling electricity back to the grid. The price of selling electricity also differs on an hourly basis, depending on the regional situation in the grid. In the case of low availability of electric power, the plant can use the possibility to decrease its internal demand, to use the negotiated contracts and to use onsite generation in order to sell the electricity with a profit. This might happen especially in regions with heavy industry and at low temperatures during winter time.

The electricity bill, apart from the electricity purchase costs, includes also deviation penalties. The plant is assumed to predict its load consumption for a period of $24 \mathrm{~h}$ minimum one day before the actual load occurs. This forecast is sent to the energy supplier, committing the plant to a certain load profile. If the actual consumption differs from this profile, the plant is obliged to pay penalties. Here, we assume that both under- and over-consumption are penalized, but with a penalty-free tolerance margin of a few percent.

\section{Monolithic model}

The proposed MILP formulation describes a power intense steel making process that produces a set of products (heats) $p \in P$ on a set of units $m \in M$, while satisfying various operational constraints. The plant is assumed to deliver a fixed number of products that are known in advance. The power consumption is both unit and product specific. The goal is to compute a one day production schedule that minimizes the total (net) cost of electricity and the weighted starting times of the tasks (i.e. a throughput related criterion). Electricity purchase includes different options and is subject to hourly price-variations. The optimization should determine the optimal amounts to be transferred from or to the electricity sources or sinks $i \in I$ at any given time interval $s \in S$. The end of the last time slot is equal to the scheduling horizon. Penalties due to the deviation from a pre-agreed load curve are incurred when a certain penalty-free buffer is exceeded and may differ for under- and overconsumption. The electricity bill can be reduced by selling the surplus of electricity. The monolithic models are described using the notation shown in Table 1 . Additional notation introduced for the bilevel solution heuristic is given in Section 6.

Table 1 Model notation

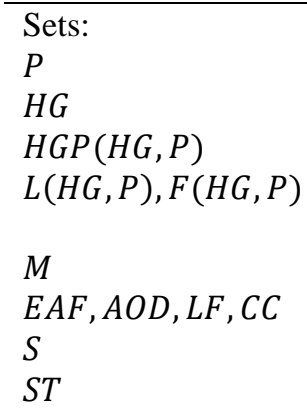

heats (products) to be produced

heat groups with defined sequence of casting subset of heats p mapped to corresponding heat group hg subset of heats $p$ cast respectively last or first in a heat group casting sequence hg equipment (machines) subsets of equipment time intervals production stage 


\begin{tabular}{|c|c|}
\hline$S M(S T, M)$ & production stage st mapped to corresponding equipment m \\
\hline Node, $I, J$ & nodes in flow network denoting sources and sinks of electricity \\
\hline Pur (Node) & purchase contracts node \\
\hline $\operatorname{Dem}($ Node $)$ & production process electricity demand node \\
\hline Gen(Node) & onsite generation node \\
\hline Bal(Node) & balancing node \\
\hline Sale (Node) & electricity sale sink node \\
\hline$A R C_{i, j, s}$ & defined arc between nodes $\mathrm{i}$ and $\mathrm{j}$ in time slot $\mathrm{s}$ \\
\hline \multicolumn{2}{|l|}{ Parameters: } \\
\hline$\theta_{p, m}$ & processing duration of heat $\mathrm{p}$ on equipment $\mathrm{m}$ \\
\hline$t_{m}^{\text {setup }}$ & setup time for machine $\mathrm{m}$ \\
\hline$t_{m, m^{\prime}}^{m i n}$ & minimum transport time from equipment $\mathrm{m}$ to $\mathrm{m}$ ' \\
\hline$t_{p, s t}^{\max }$ & maximum hold-up (waiting) time after stage st \\
\hline$a_{s}$ & pre-agreed (committed) load curve \\
\hline$\tau_{s}$ & electricity consumption time slot boundary \\
\hline$h_{p, m}$ & specific power consumption of processing heat $\mathrm{p}$ on equipment $\mathrm{m}$ \\
\hline$c_{s, i, j}$ & electricity cost of flow from $\mathrm{i}$ to $\mathrm{j}$ in time slot $\mathrm{s}$ \\
\hline$f_{s, i, j}^{\min }, f_{s, i, j}^{\max }$ & minimum and maximum flow between nodes $\mathrm{i}$ and $\mathrm{j}$ \\
\hline$r^{\min }, d^{\min }$ & minimum run- and down-time of onsite generation \\
\hline$c^{\text {start }}$ & startup cost of onsite generation \\
\hline$k$ & $\begin{array}{l}\text { coefficient of delivered power reduction due to startup of onsite } \\
\text { generation }\end{array}$ \\
\hline$c$ & coefficient of task start time weight in the objective function \\
\hline \multicolumn{2}{|l|}{ Variables: } \\
\hline$t_{m, p}^{S}, t_{m, p}^{f}$ & $\begin{array}{l}\text { positive continuous variables of starting and finishing time of heat } \mathrm{p} \\
\text { on equipment } \mathrm{m}\end{array}$ \\
\hline$t_{p, s t}^{s}, t_{p, s t}^{f}$ & $\begin{array}{l}\text { positive continuous variables of starting and finishing time of heat } \mathrm{p} \\
\text { at stage st }\end{array}$ \\
\hline$w_{p, s t}$ & positive continuous variables of waiting time of heat $\mathrm{p}$ after stage st \\
\hline$q_{s}$ & positive continuous variables of electricity consumed in time slot s \\
\hline$X_{m, p}$ & $\begin{array}{l}\text { binary variable, true when heat } \mathrm{p} \text { is assigned for processing on } \\
\text { equipment } \mathrm{m}\end{array}$ \\
\hline$V_{s t, p, p^{\prime}}$ & $\begin{array}{l}\text { binary variable, true when heat p' is processed after heat p on stage } \\
\text { st }\end{array}$ \\
\hline$Y_{p, s t, s}^{s}, Y_{p, s t, s}^{f}$ & $\begin{array}{l}\text { binary variable, true when heat p starts or finishes on stage st in the } \\
\text { slot s }\end{array}$ \\
\hline$G_{s, i, j}$ & binary variable, true when generation is running in time slot s \\
\hline$g_{s, i, j}^{s}$ & $\begin{array}{l}\text { pseudo-continuous positive variable denoting if onsite generation } \\
\text { start-up occurred in time slot s }\end{array}$ \\
\hline$y_{p, m, s t, s}^{\operatorname{saux}}, y_{p, m, s t, s}^{\text {faux }}$ & $\begin{array}{l}\text { auxiliary continuous positive variable true when heat } \mathrm{p} \text { is assigned } \\
\text { for processing and started or finished processing on stage st in time } \\
\text { slot s }\end{array}$ \\
\hline$a_{p, m, s t, s}, b_{p, m, s t, s}, c_{p, m, s t, s}, d_{p, m, s t, s}$ & $\begin{array}{l}\text { positive continuous variables accounting for processing time of heat } \\
\text { p on equipment } m \text { on stage st spent within a slot } s\end{array}$ \\
\hline$b_{s}$ & $\begin{array}{l}\text { positive continuous variables of buffer level for allowed deviation } \\
\text { from committed load in time slot s }\end{array}$ \\
\hline$b_{s}^{o}, b_{s}^{u}$ & $\begin{array}{l}\text { positive continuous variables of upper and lower bounds for buffer in } \\
\text { time slot s }\end{array}$ \\
\hline$c_{s}^{o}, c_{s}^{u}$ & $\begin{array}{l}\text { positive continuous variables of actual over- and under-consumption } \\
\text { in time slot s }\end{array}$ \\
\hline$f_{s, i, j}$ & positive continuous variables of flow from node $\mathrm{i}$ to $\mathrm{j}$ in time slot $\mathrm{s}$ \\
\hline$c_{s}^{g e n}$ & positive continuous variables of cost of onsite generation in slot s \\
\hline$\mu$ & continuous variable of net electricity consumption cost \\
\hline$\delta$ & positive continuous variables of deviation penalties cost \\
\hline
\end{tabular}

\subsection{Structure of the monolithic model}


For the problem described in Section 3 we describe a monolithic model in this section. It consists of several components that are shown in Figure 4. First, to ensure that all process specific constraints are satisfied, a scheduling model was created using the continuous-time general precedence approach (Section 4.2). The use of this approach is motivated by the required level of precision stemming from the specification by the industrial end-user. In order to optimize the purchase of electricity and to augment the schedule in order to express potential changes of load pattern, a strategy for expanding the scheduling model with energy-awareness was formulated (Section 4.3). This part of the monolithic model uses the continuous variable (used in the scheduling part) of task start time $t_{m, p}^{s}$ in order to find the contribution of a task to the electricity consumption within a given time interval $s$.

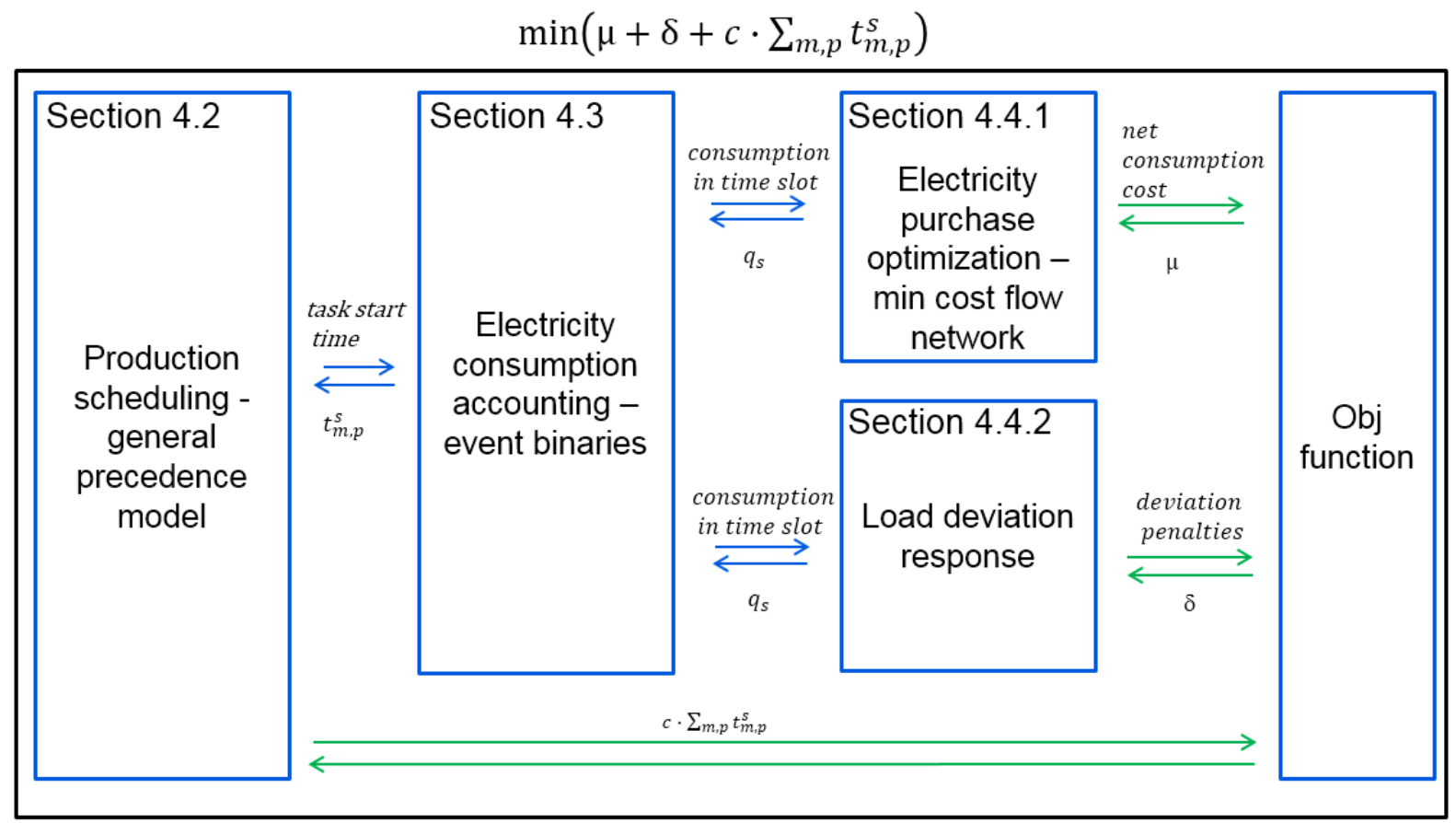

Figure 4 Monolithic model structure

When applying this strategy for all tasks, the total electricity consumption $q_{s}$ of the process in a given time interval can be computed, which is needed for the optimization of the cost of electricity (Section 4.4). This part computes optimal values in a flow network representing possible flows of electricity $f_{s, i, j}$ from sources to sinks. The optimization results in an optimal cost structure of the available purchase contracts with the exact amount of the electricity to be bought or sold under each contract. The knowledge of the process consumption during the time slots also enables to account for potential penalties $\delta$ paid due to deviations from pre-agreed load curve, and to determine when it is profitable to under- or over-consume electricity.

The objective function of the monolithic model takes into account the weighted task start times, the net electricity consumption cost $\mu$ and penalties $\delta$ paid for deviations. By choosing the weights in the summation, potential losses in the process (e.g. heat losses due to waiting time between the stages) or delays of the production can be traded off against the cost of electricity purchase and sales.

\subsection{Production scheduling model}

The general precedence scheduling model for the stainless-steel plant used in this study is largely based on the model introduced by Harjunkoski and Grossmann (2001). This model was further 
extended to a more flexible formulation introducing stages and multiple machines in Harjunkoski and Sand (2008). The scheduling part of the model uses assignment and precedence binaries following equations (1-17) from Hadera and Harjunkoski (2013).

The scheduling model is based on the precedence variables and assignment variables that determine which of the parallel machines on each stage shall process a given heat. The general precedence $V_{s t, p, p^{\prime}}$ is true if a product $p$ is processed before a product $p^{\prime}$ on a stage $s t$. The assignment $X_{m, p}$ is true only when a given product $p$ is processed on machine $m$. The sum (Eq. 1) states that exactly one machine should process a heat per stage.

$\sum_{m \in S M_{s t, m}} X_{m, p}=1 \quad \forall p \in P, s t \in S T$

Equation (2) defines the finishing time $t_{m, p}^{f}$ as the starting time $t_{m, p}^{s}$ plus the selected processing length $\theta_{p, m}$.

$t_{m, p}^{f}=t_{m, p}^{s}+X_{m, p} \cdot \theta_{p, m} \quad \forall m \in M, p \in P$

Since a product can be processed only once on a given machine, the unassigned machines get a zero starting time (Eq. 3).

$t_{m, p}^{S} \leq M \cdot X_{m, p} \quad \forall m \in M, p \in P$

The stage starting and finishing times $t_{p, s t}^{s}, t_{p, s t}^{f}$ are synchronized with the corresponding machine times in Eqs. (4-5).

$t_{p, s t}^{S}=\sum_{m \in S M_{s t, m}} t_{m, p}^{S} \quad p \in P, s t \in S T$

$t_{p, s t}^{f}=\sum_{m \in S M_{s t, m}} t_{m, p}^{f} \quad p \in P, s t \in S T$

The scheduling model handles maximum hold-up times after processing has been completed on a given stage, equipment specific setup $t_{m}^{\text {setup }}$ times and minimum transportation times. The processing on the next stage can be done only after the processing of the previous stage has finished plus some waiting time $w_{p, s t}$, which serves here as a slack variable which is determined by the optimization. The production flow between subsequent stages is established in Eq. (6).

$t_{p, s t+1}^{s}=t_{p, s t}^{f}+w_{p, s t} \quad \forall p \in P, s t \in S T, s t<|S T|$

Due to process restrictions, it is necessary to enforce lower and upper bounds for the waiting times. The minimum corresponds to the physical possibility of transferring the product to the next stage, and it is equal to the minimum transportation time between machines $t_{m, m^{\prime}}^{\min }$ as stated in (7). The upper bound $t_{p, s t}^{\max }$ of the waiting time reflects the process constraint that a heat should not cool off below a certain level.

$t_{m, m^{\prime}}^{\min }\left(X_{m, p}+X_{m^{\prime}, p}-1\right) \leq w_{p, s t} \leq t_{p, s t}^{\max }$

$\forall p \in P, m, m^{\prime} \in M$, st $\in S T,\{s t, m\} \in S M,\left\{s t+1, m^{\prime}\right\} \in S M, \quad s t<|S T|$

The precedence of the products is characterized by the fact that either $p$ is processed after $p^{\prime}$ or $p^{\prime}$ is processed after $p$. Therefore, only one of the two binaries can be true. Equation (8) enforces a correct sequencing. 
$V_{s t, p, p^{\prime}}+V_{s t, p^{\prime}, p}=1 \quad \forall p, p^{\prime} \in P, s t \in S T, p<p^{\prime}$

In order to impose the common practice that the sequence of the products that are casted on a CC must propagate back to the other production stages, Eq. (9) is introduced.

$V_{s t, p, p^{\prime}}=V_{s t+1, p, p^{\prime}} \quad \forall p, p^{\prime} \in P, s t \in S T, p<p^{\prime}, s t<|S T|$

The precedence constraint in (10) for other stages than CC restricts that a next heat should be processed only after the previous one has finished plus a setup time.

$t_{m, p^{\prime}}^{s} \geq t_{m, p}^{f}+t_{m}^{\text {setup }}-\left(M+t_{m}^{\text {setup }}\right)\left(3-V_{s t, p, p^{\prime}}-X_{m, p}-X_{m, p^{\prime}}\right)$

$\forall p, p^{\prime} \in P, m \in M$, st $\in S T,\{s t, m\} \in S M, p \neq p^{\prime}, s t<|S T|$

At the CC-stage no setup time $t_{m}^{\text {setup }}$ should occur to ensure continuous casting (Eq. 11). However, a setup must be carried out between the last $L(H G, P)$ and first $F(H G, P)$, heats of different heat groups (Eq. 12).

$t_{m, p^{\prime}}^{s} \geq t_{m, p}^{f}-M\left(3-V_{s t, p, p^{\prime}}-X_{m, p}-X_{m, p^{\prime}}\right)$

$\forall p, p^{\prime} \in P, m \in M$, st $\in S T,\{s t, m\} \in S M, p \neq p^{\prime}, s t=|S T|$

$t_{m, p^{\prime}}^{s} \geq t_{m, p}^{f}+t_{m}^{\text {setup }}-\left(M+t_{m}^{\text {setup }}\right)\left(3-V_{s t, p, p^{\prime}}-X_{m, p}-X_{m, p^{\prime}}\right)$

$\forall p \in L(H G, P), p^{\prime} \in F(H G, P), m \in M, s t \in S T,\{s t, m\} \in S M, p \neq p^{\prime}, s t=|S T|$

Constraint (13) ensures that heats of the same heat group are assigned to the same caster.

$X_{m, p}=X_{m, p+1} \quad \forall p \in P, m \in M, h g \in H G,\{h g, p\} \in H G P(P),\{s t, m\} \in S M, s t=|S T|$

As the heats are pre-ordered within a casting sequence, Eq. (14) ensures that next heat in a sequence starts immediately after the previous one has finished.

$t_{p+1, s t}^{s}=t_{p, s t}^{f} \quad \forall p \in P \backslash L(H G, P)$, st $\in S T, s t=|S T|$

From technical process requirements, the heat sequence within one heat group is known. The precedence of heats within one heat group is enforced and redundant values are eliminated in Eq. (15). Redundant sequencing variables are eliminated when comparing two identical products in Eq. (16).

$V_{s t, p, p^{\prime}}=1 \quad \forall p, p^{\prime} \in P, p<p^{\prime}$, st $\in S T, h g \in H G,\{h g, p\},\left\{h g, p^{\prime}\right\} \in H G P(P)$

$V_{s t, p, p^{\prime}}=0 \quad \forall p, p^{\prime} \in P$, st $\in S T, p=p^{\prime}$

Since the goal of the production plant is to meet the production targets as soon as possible, minimizing the makespan (or tasks completion time) can be specified as an objective function in the MILP model.

\subsection{Energy-awareness in precedence based scheduling}

In continuous-time models, it is challenging to account for resource consumption. In this work we extend the scheduling model described above to account for the electricity consumption by each task within given time intervals of interest. The time grid with intervals in our use case corresponds to 
volatile electricity prices and committed load values. Therefore the length of the intervals is one hour. The scheduling model uses continuous task start time variables which are linked to the energy-aware part of the model, leading to the computation of the overall electricity consumption within a given time interval. Once the model is complemented by energy-awareness, both the electricity purchase and the load commitment can be optimized.

\subsubsection{Model with event binaries}

They key idea in the approach developed here is the use of the two event binaries representing whether a given task started $\left(Y_{p, s t, s}^{s}\right)$ or finished $\left(Y_{p, s t, s}^{f}\right)$ in or before or after particular time slot $s$ (Figure 5).

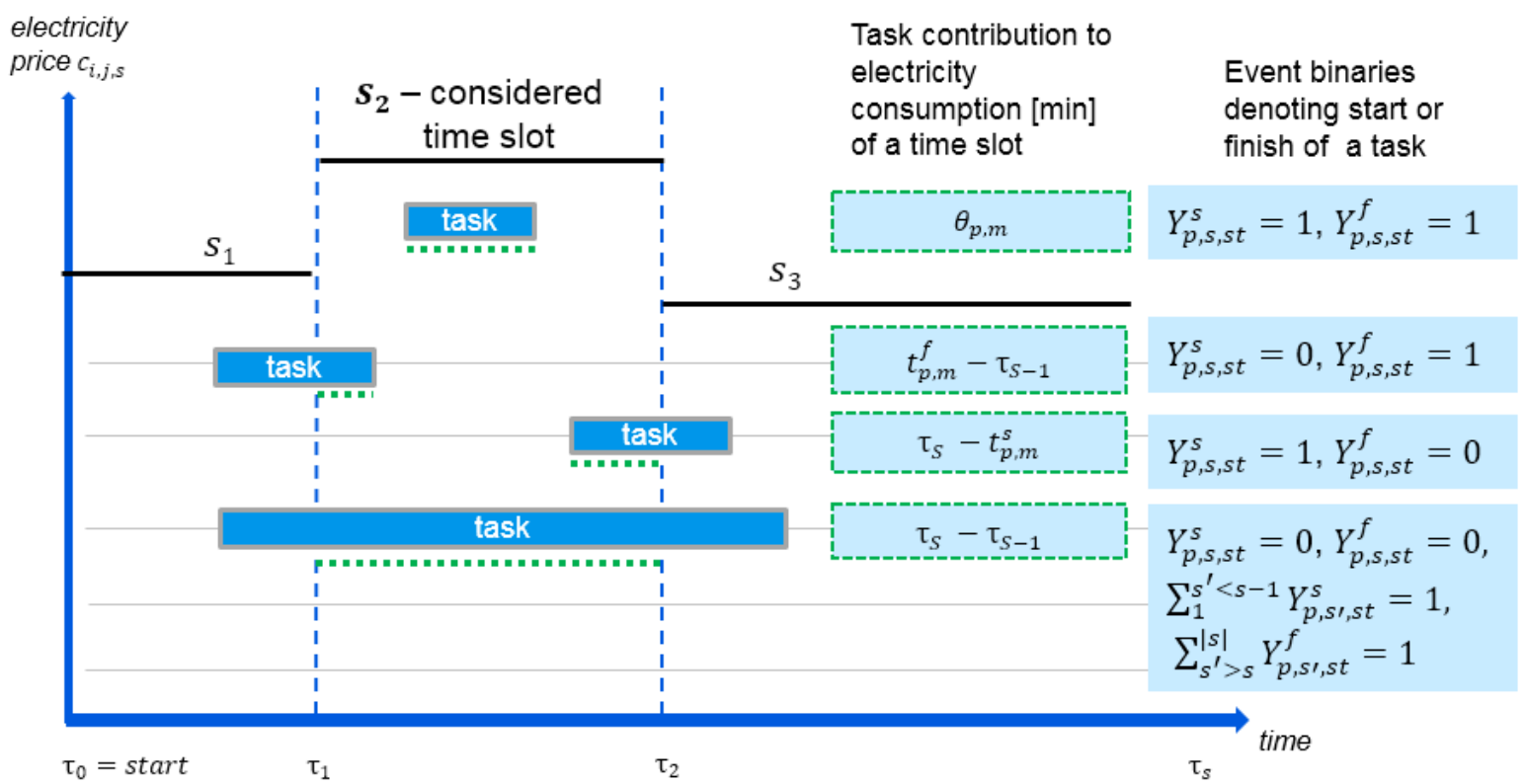

Figure 5 Event binaries model to describe the consumption of electric energy in time slots of the price

Since the boundaries of the time slot $s$ are known, Big-M constraints in Eqs. (17-20) force the event binaries to be true in case the start or finish variable takes a value between the time slot's upper bound $\tau_{s}$ and lower bound $\tau_{s-1}$.

$$
\begin{aligned}
& t_{p, s t}^{s} \geq \tau_{s-1} \cdot Y_{p, s t, s}^{s} \quad \forall p \in P, s t \in S T, s \in S(17) \\
& t_{p, s t}^{s} \leq \tau_{s}+\left(M-\tau_{s}\right)\left(1-Y_{p, s t, s}^{s}\right) \quad \forall p \in P, s t \in S T, s \in S \\
& t_{p, s t}^{f} \geq \tau_{s-1} \cdot Y_{p, s t, s}^{f} \quad \forall p \in P, s t \in S T, s \in S \\
& t_{p, s t}^{f} \leq \tau_{s}+\left(M-\tau_{s}\right)\left(1-Y_{p, s t, s}^{f}\right) \quad \forall p \in P, s t \in S T, s \in S
\end{aligned}
$$

However, the use of the stage set st in the definition of the event binaries does not indicate which of the available equipment of this stage is actually processing. Therefore, together with the assignment variable $X_{p, m}$ we can introduce two additional auxiliary pseudo-binary variables $y_{p, m, s t, s}^{\text {saux }}$ and $y_{p, m, s t, s}^{\text {faux }}$ that will be true only in case the respective event binary is true and the assignment is true as well (Eqs. 21-26). 


$$
\begin{aligned}
& y_{p, m, s t, s}^{\operatorname{saux}} \geq X_{m, p}+Y_{p, s t, s}^{s}-1 \quad \forall p \in P, m \in M, s t \in S T, s \in S,\{s t, m\} \in S M \\
& y_{p, m, s t, s}^{\text {saux }} \leq X_{m, p} \quad \forall p \in P, m \in M, s t \in S T, s \in S,\{s t, m\} \in S M \\
& y_{p, m, s t, s}^{\text {saux }} \leq Y_{p, s t, s}^{s} \quad \forall p \in P, m \in M, s t \in S T, s \in S,\{s t, m\} \in S M \\
& y_{p, m, s t, s}^{\text {faux }} \geq X_{m, p}+Y_{p, s t, s}^{f}-1 \quad \forall p \in P, m \in M, s t \in S T, s \in S,\{s t, m\} \in S M \\
& y_{p, m, s t, s}^{\text {faux }} \leq X_{m, p} \quad \forall p \in P, m \in M, s t \in S T, s \in S,\{s t, m\} \in S M \\
& y_{p, m, s t, s}^{\text {faux }} \leq Y_{p, s t, s}^{f} \quad \forall p \in P, m \in M, s t \in S T, s \in S,\{s t, m\} \in S M
\end{aligned}
$$

The two auxiliary binaries will have indices representing product $p$, machine $m$, stage $s t$ and time slot $s$. That enables us to introduce continuous variables that are used to capture different cases of how a particular task (here a heat processed on a unit) relates to a time slot. As shown in Figure 5 there are four different scenarios:

\section{A task is processed entirely within the time slot}

Processing within a time slot means that the start and finish time of the task must be placed within the time slot upper and lower boundary, both event binaries need to hold true. To capture this case we introduce an auxiliary variable $a_{p, m, s t, s}$ described in Equation (27-29).

$$
\begin{aligned}
& a_{p, m, s t, s} \geq y_{p, m, s t, s}^{\text {saux }}+y_{p, m, s t, s}^{\text {faux }}-1 \quad \forall p \in P, m \in M, s t \in S T, s \in S,\{s t, m\} \in S M \\
& a_{p, m, s t, s} \leq y_{p, m, s t, s}^{\operatorname{saux}} \quad \forall p \in P, m \in M, s t \in S T, s \in S,\{s t, m\} \in S M \\
& a_{p, m, s t, s} \leq y_{p, m, s t, s}^{\text {faux }} \quad \forall p \in P, m \in M, s t \in S T, s \in S,\{s t, m\} \in S M
\end{aligned}
$$

\section{A task starts before and finishes within the time slot}

For this case the start binary shall be zero for the considered slot. However the finish binary must hold true. That combination of the two binaries is enough to capture the time contribution $b_{p, m, s t, s}$ of the task, as shown in Equation (30-33).

$$
\begin{aligned}
& b_{p, m, s t, s} \geq t_{p, m}^{f}-\tau_{s-1}-\left(M-\tau_{s-1}\right)\left(1-y_{p, m, s t, s}^{\text {faux }}+y_{p, m, s t, s}^{\text {saux }}\right) \\
& \forall p \in P, m \in M, s t \in S T, s \in S,\{s t, m\} \in S M \\
& b_{p, m, s t, s} \leq t_{p, m}^{f}-\tau_{s-1}+\tau_{s-1}\left(1-y_{p, m, s t, s}^{f a u x}\right) \\
& \forall p \in P, m \in M, s t \in S T, s \in S,\{s t, m\} \in S M \\
& b_{p, m, s t, s} \leq\left(\tau_{s}-\tau_{s-1}\right) y_{p, m, s t, s}^{f a u x} \\
& \forall p \in P, m \in M, s t \in S T, s \in S,\{s t, m\} \in S M \\
& b_{p, m, s t, s} \leq\left(\tau_{s}-\tau_{s-1}\right)\left(1-y_{p, m, s t, s}^{s a u x}\right) \\
& \forall p \in P, m \in M, s t \in S T, s \in S,\{s t, m\} \in S M
\end{aligned}
$$




\section{A task starts within and finishes after the time slot}

In this case, the task should start between the lower and the upper bounds of the time slot and finish sometime after the upper bound. That means the start event binary is true for the slot and the finish event binary is false. The variable $c_{p, m, s t, s}$ is defined by Equation (34-37).

$c_{p, m, s t, s} \geq \tau_{s}-t_{p, m}^{s}-\tau_{s}\left(1-y_{p, m, s t, s}^{\text {saux }}+y_{p, m, s t, s}^{\text {faux }}\right)$

$\forall p \in P, m \in M, s t \in S T, s \in S,\{s t, m\} \in S M$

$c_{p, m, s t, s} \leq \tau_{s}-t_{p, m}^{s}+\left(M-\tau_{s}\right)\left(1-y_{p, m, s t, s}^{\text {saux }}\right)$

$\forall p \in P, m \in M, s t \in S T, s \in S,\{s t, m\} \in S M$

$c_{p, m, s t, s} \leq\left(\tau_{s}-\tau_{s-1}\right) y_{p, m, s t, s}^{\text {saux }}$

$\forall p \in P, m \in M, s t \in S T, s \in S,\{s t, m\} \in S M$

$c_{p, m, s t, s} \leq\left(\tau_{s}-\tau_{s-1}\right)\left(1-y_{p, m, s t, s}^{\text {faux }}\right)$

$\forall p \in P, m \in M, s t \in S T, s \in S,\{s t, m\} \in S M$

4. A task over-spans the full time slot

This occurs only when the start time of the task is placed before the lower bound of the time slot and at the same time the finish time of the task occurs after the upper bound of the time slot. This translates into zero values for both of the event binaries. In addition the start event binary is true in one of the earlier slots before the considered one, and similarly, the finish event binary is true in one of the later time slots. The variable $d_{p, m, s t, s}$ is defined by the constraints in Equations (38-42). If the task either started or finished entirely before the considered slot or after the slot it does not contribute to the electricity consumption within the slot.

$$
\begin{aligned}
& d_{p, m, s t, s} \geq\left(\tau_{s}-\tau_{s-1}\right) \cdot\left(\sum_{1}^{s^{\prime}<s} y_{p, m, s t, s^{\prime}}^{\text {saux }}+\sum_{s^{\prime}>s}^{|S|} y_{p, m, s t, s^{\prime}}^{\text {faux }}-1\right) \\
& \forall p \in P, m \in M, s t \in S T, s \in S,\{s t, m\} \in S M \\
& d_{p, m, s t, s} \leq\left(\tau_{s}-\tau_{s-1}\right) \cdot \sum_{1}^{s^{\prime}<s} y_{p, m, s t, s^{\prime}}^{\text {saux }} \quad \forall p \in P, m \in M, s t \in S T, s \in S,\{s t, m\} \in S M \\
& d_{p, m, s t, s} \leq\left(\tau_{s}-\tau_{s-1}\right) \cdot \sum_{s^{\prime}>s}^{|S|} y_{p, m, s t, s^{\prime}}^{f a u x} \quad \forall p \in P, m \in M, s t \in S T, s \in S,\{s t, m\} \in S M \\
& d_{p, m, s t, s} \leq\left(\tau_{s}-\tau_{s-1}\right)\left(1-y_{p, m, s t, s}^{\text {saux }}\right) \quad \forall p \in P, m \in M, s t \in S T, s \in S,\{s t, m\} \in S M \\
& d_{p, m, s t, s} \leq\left(\tau_{s}-\tau_{s-1}\right)\left(1-y_{p, m, s t, s \prime}^{f a u x}\right) \quad \forall p \in P, m \in M, s t \in S T, s \in S,\{s t, m\} \in S M
\end{aligned}
$$

The constraints based on the above cases yield the continuous variables $b_{p, m, s t, s}, c_{p, m, s t, s}, d_{p, m, s t, s}$ accounting for how much time a given processing task spends within the considered time slot. Since the specific electricity consumption of the processing task is known, a proper summation of a product of the continuous variables and machine-specific electricity consumption parameter accounts for the total consumption in a given time slot (Eq. 43). The above described approach yields fewer binaries 
than the one used by Nolde and Morari (2010), where six binary variables are used to describe the relation between the task and the time slot. Here only two event binaries are needed.

$q_{s}=\left(\sum_{p \in P, m \in M} h_{p, m}\left(a_{p, s, s t, m} \tau_{p, m}+b_{p, s, s t, m}+c_{p, s, s t, m}+d_{p, s, s t, m}\right)\right) / 60 \quad \forall s \in S$

A set of tightening constraints can help to speed up the computational performance of the model. In equations (44) and (45) we restrict that for only one slot within the entire time horizon the event binary is active. Additionally, it is true only when a task exist, i.e. when a product is assigned to be processed on a machine. Equation (46) accounts for total consumption of the schedule to be equal to sum of total consumption of those tasks that has been assigned.

$\sum_{s \in S} Y_{p, s t, s}^{s}=\sum_{m \in M,\{s t, m\} \in S M} X_{m, p} \quad \forall p \in P, s t \in S T$

$\sum_{s \in S} Y_{p, s t, s}^{f}=\sum_{m \in M,\{s t, m\} \in S M} X_{m, p} \quad \forall p \in P, s t \in S T$

$\left(\sum_{p \in P, m \in M} X_{m, p} \cdot h_{p, m} \cdot \pi_{p, m}\right) / 60=\sum_{s \in S} q_{s}$

\subsubsection{Literature model}

Other formulations of resource consumption accounting in continuous-time based scheduling models have already been reported in the literature. Nolde and Morari (2010) presented a formulation that introduces six binaries to capture six different cases of the position a task might have relative to a time interval on the time axis. This approach was later reformulated by Hadera and Harjunkoski (2013) to account for parallel machines at each production stage with the goal of optimizing the cost of electricity for a single price curve and load deviation penalties. To reduce the model size, starting and finishing times of tasks were replaced by the corresponding stage starting and finishing times. The resulting model formulation is presented in Appendix 1 and is later used in the computations (Section 5.3) to compare its performance to the event binaries formulation described in Section 4.3.1.

\subsection{Optimization of the cost of electricity}

\subsubsection{Multiple purchase sources optimization}

The tracking of the consumption of electricity over the time intervals can be used for optimizing the purchase and sales strategy. Once the scheduling model has been extended by the corresponding values of electricity consumption in the time slots, the purchase optimization can influence the schedule in such way that a mixed criterion (with e.g. task start times as used later) that includes also the cost of electricity is minimized. The idea for purchase optimization is based on a minimum cost flow network formulation (Figure 6) with a balancing node for which all inflows are equal to all outflows (Eq. 47). The inflow nodes represent the possible sources of electricity. The outflow nodes are the process demand and the selling of electricity.

$\sum_{i \in \text { Node }} f_{s, i, j^{\prime}}=\sum_{j \in N o d e} f_{s, j^{\prime}, j} \quad \forall\left(i, j^{\prime}\right),\left(j^{\prime}, j\right) \in A r c, j^{\prime} \in B a l, s \in S$ 


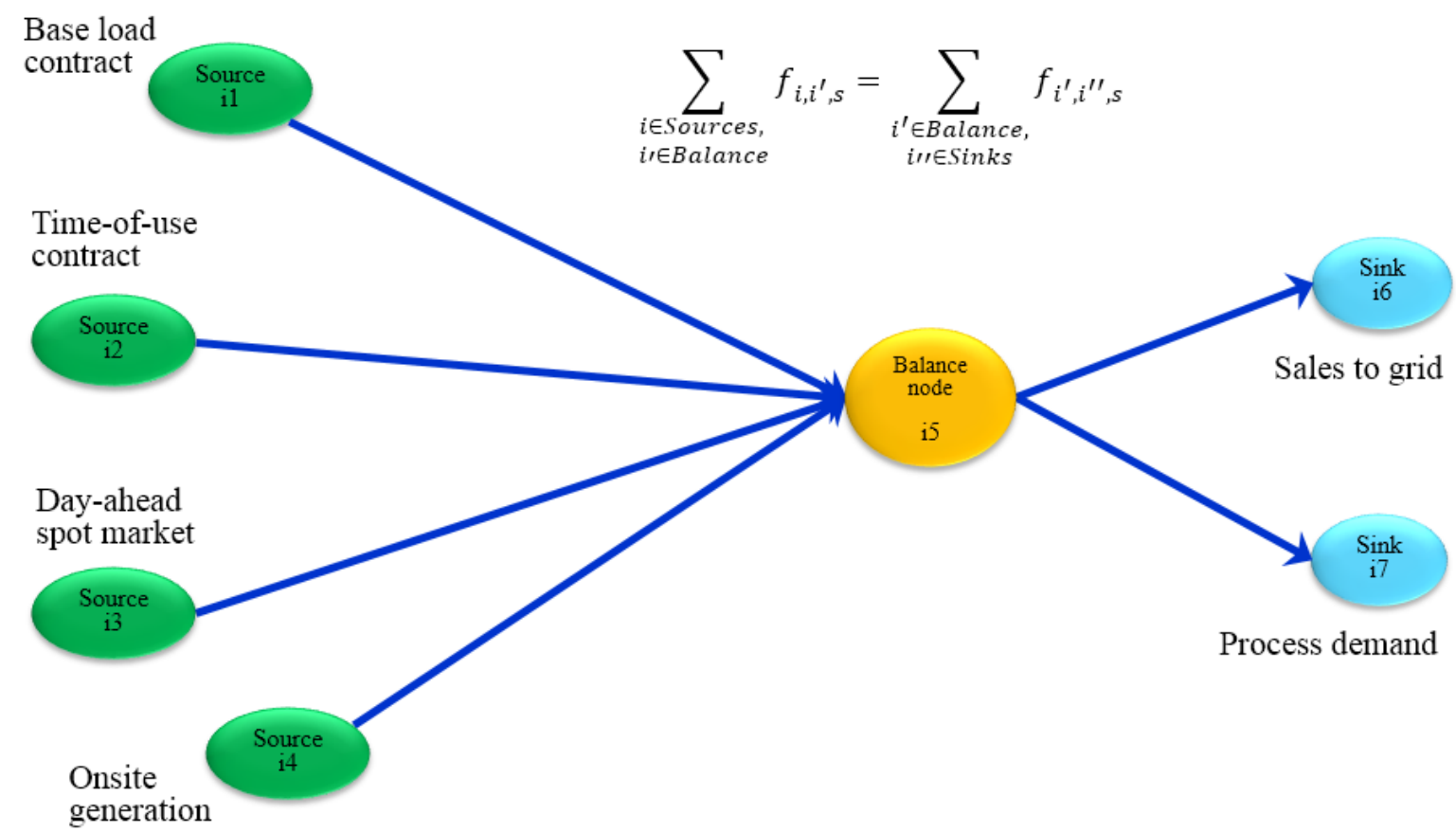

Figure 6 Formulation of the electricity purchase and sale optimization problem

The balancing node is connected with the sink and the source nodes by arcs that are characterized by parameters and variables. An arc exists only if there is a cost defined for it. The parameters are the minimum and maximum levels of the flows between two given nodes (Eq. 48) and the cost function.

$f_{s, i, j}^{\min } \leq f_{s, i, j} \leq f_{s, i, j}^{\max } \quad \forall(i, j) \in$ Arc $, i, j \in$ Node, $s \in S$

The network is used to identify the most economical flows while satisfying the load from the process demand node (Eq. 49).

$\mathrm{q}_{\mathrm{s}}=\sum_{\mathrm{i} \in \text { Node, }, \in \text { Dem }} \mathrm{f}_{\mathrm{s}, \mathrm{i}, \mathrm{j}} \quad \forall(i, j) \in \operatorname{Arc}, s \in S$

The onsite generation is modelled using a binary variable $G_{s, i, j}$ that denotes whether the plant is in production mode (Eq. 50) and an auxiliary pseudo-continuous variable $g_{s, i, j}^{s}$ indicating generation start-up (Eqs. 51-52). Here, the Big-M value $M_{2}$ should not be less than the maximum flow on the arc between the onsite generation and the balancing node.

$G_{s, i, j} \leq f_{s, i, j} \leq M_{2} \cdot G_{s, i, j} \quad \forall(i, j) \in$ Arc, $i \in$ Gen, $j \in$ Bal, $s \in S$

$G_{s, i, j}-G_{s-1, i, j} \leq g_{s, i, j}^{s} \leq G_{s, i, j} \quad \forall(i, j) \in$ Arc, $i \in$ Gen, $j \in$ Bal, $s \in S$

$0 \leq g_{s, i, j}^{s} \leq 1-G_{s-1, i, j} \quad \forall(i, j) \in$ Arc, $i \in$ Gen, $j \in$ Bal, $s \in S$

The onsite generation constraints are kept simple by considering a constant generation cost with additional start-up cost (Eq. 53) and a reduced production rate by a factor $k$ for those time intervals where a start-up occurs (Eq. 54).

$c_{\mathrm{s}}^{\text {gen }}=\sum_{\mathrm{i} \in \text { Node }, j \in G e n} \mathrm{f}_{\mathrm{s}, \mathrm{i}, \mathrm{j}} \cdot \mathrm{c}_{\mathrm{s}, \mathrm{i}, \mathrm{j}}+\mathrm{c}^{\text {start }} \cdot \mathrm{g}_{\mathrm{s}, \mathrm{i}, \mathrm{j}}^{\mathrm{s}} \quad \forall(i, j) \in \operatorname{Arc}, s \in S$

$f_{s, i, j}=f_{s, i, j}^{\max } \cdot G_{s, i, j}-k \cdot f_{s, i, j}^{\max } \cdot g_{s, i, j}^{s} \quad \forall(i, j) \in$ Arc $, i \in G e n, j \in$ Bal, $s \in S$ 
Moreover, a minimum runtime $r^{\text {min }}$ and a minimum downtime $d^{\text {min }}$ are enforced (Eqs. 55-56). The implementation of more detailed constraints that are available in literature would also be possible here, including accounting for steam flows and more detailed electricity production rates as for example in Mitra et al. (2013).

$\sum_{S^{\prime}=s}^{s+r^{\min }-1} G_{s^{\prime}, i, j} \geq r^{\min }\left(G_{s, i, j}-G_{s-1, i, j}\right)$

$\forall(i, j) \in$ Arc,$i \in$ Gen, $j \in$ Bal, $s \in S, s<|S|-r^{\text {min }}$

$\sum_{S^{\prime}=s}^{s+d^{\min }-1} G_{s^{\prime}, i, j} \leq d^{\min }\left(1+G_{s, i, j}-G_{s-1, i, j}\right)$

$\forall(i, j) \in$ Arc, $i \in$ Gen, $j \in$ Bal, $s \in S, s<|S|-d^{\text {min }}$

The final net electricity purchase cost (Eq. 57) is composed of the cost associated with purchase from contracts, the cost of the generation and the revenues from the electricity sold.

$\mu=\sum_{s \in S}\left(\sum_{i \prime \in N o d e, j \prime \in P u r} f_{s, i \prime, j} \cdot c_{s, i, j}+c_{s}^{g e n}-\sum_{i \in \text { Node }, j \in S a l e} f_{s, i, j} \cdot c_{s, i, j}\right)$

$\forall(i, j),\left(i^{\prime}, j^{\prime}\right) \in \operatorname{Arc}$

\subsubsection{Load deviation problem}

As a steel plant is a large consumer, the suppliers of electricity impose that it commits to certain hourly varying levels of load. We only consider here the situation where there is only one supplier and the committed load is the total load of the steel plant. Instead it could also be the load that is covered by one of the contracts, or there could be several such curves. In case the actual consumption deviates from the pre-agreed values, financial penalties are incurred. The part of the model that accounts for the penalties is similar to the formulation in Hadera and Harjunkoski (2013). For the load tracking error penalties, it is assumed that there is a penalty-free deviation (buffer) $b_{s}$ that is relative to the committed consumption $a_{s}$ and limited by relative upper and lower bounds $b_{s}^{o}$ and $b_{s}^{u}$ as stated in Eq. (58).

$-a_{s} \cdot b_{s}^{u} \leq b_{s} \leq a_{s} \cdot b_{s}^{o} \quad \forall s \in S$

The actual levels of over- and under consumption ( $c_{s}^{o}$ and $\left.c_{s}^{u}\right)$ are determined by Eq. (59).

$q_{s}=a_{s}+c_{s}^{o}-c_{s}^{u}+b_{s} \quad \forall s \in S$

The penalty term $\delta$ calculating the fines $p^{o}, p^{u}$ for over- and under consumption is given by Eq. (60).

$\delta=p^{o} \cdot \sum_{s \in S} c_{S}^{o}+p^{u} \cdot \sum_{s \in S} c_{S}^{u}$

The final objective function of the monolithic model in Eq. (61) minimizes the net electricity cost $\mu$, the deviation penalties and the weighted sum of the task starting times $t_{p, m}^{s}$ with $c$ being a weighting factor.

$\min \left(\mu+\delta+c \cdot \sum_{p \in P, m \in M} t_{p, m}^{s}\right)$

The part of the model that concerns the deviation problem can easily be used in load commitment of one particular contract. For example, when changing the variable representing the total consumption in a time slot $q_{s}$ to the amount drawn from Time-of-Use source $f_{s, i, j}$, where $i \in T O U$ and $j \in B a l$ we get the committed load problem of the TOU contract. 


\section{Industrial case study}

The assumptions on the constraints of the steel making process include the knowledge of the sequences and assignments of products to the last stage, the Continuous-Casting (CC) stage. We assume that it is known which products must be processed on one of the casters. However, the assignment of the heats to other units in other stages must be determined by the optimization. We also assume that the sequence of the heats that must be processed on a particular caster is known. However, it is up to the optimization to determine the sequence of those products that can be processed on two different CC machines. This assumption reduces the size of the search space. It is a reasonable assumption because very often the sequence of the products to be processed is dictated by higher level planning solutions (e.g. mill-wide planning) that are directly linked with customer orders and knowledge concerning in-house inventory levels. For integrated steel plants, the further processing of the steel slabs is carried out in the Hot Rolling Mill (HRM) after the Melt Shops section. At the HRM section it is important to define a sequence of steel slabs to be rolled such that the cost of reheating using natural gas is minimized. This challenging optimization problem of coordination between Melt Shop and Hot Rolling Mill (Xu et al. 2012) can also determine the sequence of the products on the CC stage. Usually, the assignments and the sequences on the casters reflects the quality requirements for steel, i.e. one of the casters processes certain high quality types of steel, while the other one might not be able to deliver the same qualities. The timing of the tasks to avoid production delays and to minimize the cost of energy-related problem is subject of the optimization.

\subsection{Calculation of lower and upper bounds of task start times}

To tighten the MILP model, we calculate lower and upper bounds for the task start variables. For each heat group we solve two optimization problems, minimizing and maximizing the task start time of the first product in the heat group at the CC stage. In this way we check what is the minimum value of the variable when a given heat group finishes as soon as possible on the CC. Similarly, we check what is the maximum value of the variable when a given heat group finishes as late as possible on the CC. Based on this knowledge, it is possible, using process parameters, to calculate the earliest start times and the latest start times of each task at the other stages as shown in Appendix 2. The bounds obtained from the above optimization are then propagated to the monolithic model and to the heuristic optimization in order to impose upper and lower bounds for task start and finish time variables. Finding tighter bounds helps to speed up the solution of the MILP model since then many of the energy-related binaries can be set to zero.

\subsection{Case study data}

Following the Demand Response strategy the plant has the goal of delivering a fixed number of heats (products) within a scheduling horizon of $24 \mathrm{~h}$. Due to the continuous casting requirement, products are divided within heat groups as defined in Table 2.

Table 2 Heat group definition

\begin{tabular}{cl}
\hline Group & \multicolumn{1}{c}{$\begin{array}{c}\text { Heat } \\
\text { (product) }\end{array}$} \\
\hline HG1 & P1-P3 \\
HG2 & P4-P7 \\
HG3 & P8-P12 \\
HG4 & P13-P16 \\
HG5 & P17-P20 \\
\hline
\end{tabular}


For the test cases with fewer products the last heats were excluded. Processing times and specific electricity consumption of the tasks are given in Table 3, while setup times are reported in Table 4 . Minimum transportation times and maximum waiting (hold-up) times after processing on a given stage are shown in Table 5 and Table 6 respectively.

Table 3 Processing times and electricity consumption

\begin{tabular}{ccccc}
\hline & EAF1, EAF2 & AOD1, AOD2 & LF1, LF2 & CC1, CC2 \\
\cline { 2 - 5 } P1-P20 & $85[\mathrm{~min}]$ & $8[\mathrm{~min}]$ & $45[\mathrm{~min}]$ & $60[\mathrm{~min}]$ \\
& $85[\mathrm{MW}]$ & $2[\mathrm{MW}]$ & $2[\mathrm{MW}]$ & $7[\mathrm{MW}]$ \\
\hline
\end{tabular}

Table 4 Setup times [min]

\begin{tabular}{cc}
\hline Machine & Setup time \\
\hline EAF1, EAF2 & 9 \\
AOD1, AOD2 & 5 \\
LF1 & 15 \\
LF2 & 5 \\
CC1 & 50 \\
CC2 & 70 \\
\hline
\end{tabular}

Table 5 Transportation times [min]

\begin{tabular}{ccccccc}
\hline & AOD1 & AOD2 & LF1 & LF2 & CC1 & CC2 \\
\hline EAF1 & 10 & 25 & & & & \\
EAF2 & 25 & 10 & & & & \\
AOD1 & & & 4 & 20 & & \\
AOD2 & & & 20 & 4 & & \\
LF1 & & & & & 20 & 45 \\
LF2 & & & & & 45 & 20 \\
\hline
\end{tabular}

Table 6 Maximum waiting times after stages

\begin{tabular}{cccc}
\hline & ST1 & ST2 & ST3 \\
\cline { 2 - 4 } P1-P20 & 60 & 90 & 60 \\
\hline
\end{tabular}

The input data concerning the electricity purchase limits are shown in Figure 7. 


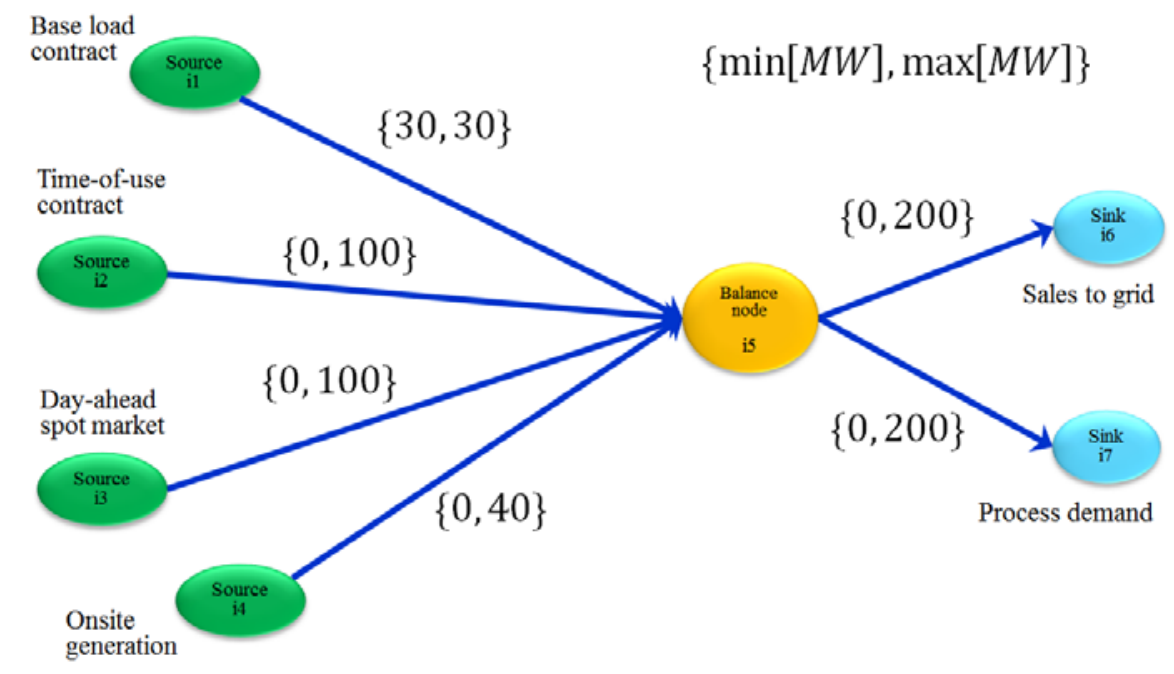

Figure 7 Bounds of the flows in the purchase flow network

Note that the base load contract has a fixed amount of delivery for each hour of the day, regardless whether the electricity is needed for the production process or not. The prices of electricity and the committed load curve are shown in Table 7Table 8. The electricity prices of both day-ahead contract cases, low price (EPEX 2013, Germany/Austria 23/09/2013) and high price (EPEX 2013, France $10 / 02 / 2012$ ) are taken from a real spot market. The pre-agreed load curve comes from a valid production schedule which was computed not considering the energy cost in the optimization, but in our case only the lead times optimization $\left(c \cdot \sum_{m \in M, p \in P} t_{m, p}^{S}\right.$, here ). This follows the previous studies (Castro et al. 2013) where the schedule with optimized production-specific cost (makespan) served as a basis for the comparison with an energy-driven schedule to assess the iDSM benefits.

Table 7 Electricity prices for case studies

\begin{tabular}{lcccc}
\hline $\begin{array}{c}\text { Time } \\
\text { interval }\end{array}$ & $\begin{array}{c}\text { Base load } \\
\text { prices } \\
\text { [€/MWh] }\end{array}$ & $\begin{array}{c}\text { Day-ahead } \\
\text { high prices } \\
\text { [€/MWh] }\end{array}$ & $\begin{array}{c}\text { Day-ahead low } \\
\text { prices } \\
\text { [€/MWh] }\end{array}$ & $\begin{array}{c}\text { TOU } \\
{[€ / M W h]}\end{array}$ \\
\hline s1 & 52 & 95 & 12,0 & 65 \\
s2 & 52 & 113 & 13,1 & 65 \\
s3 & 52 & 90 & 9,8 & 65 \\
s4 & 52 & 75 & 9,8 & 65 \\
s5 & 52 & 61 & 11,5 & 65 \\
s6 & 52 & 85 & 18,8 & 65 \\
s7 & 52 & 140 & 39,1 & 65 \\
s8 & 52 & 186 & 57,9 & 65 \\
s9 & 52 & 176 & 62,7 & 65 \\
s10 & 52 & 605 & 61,0 & 65 \\
s11 & 52 & 431 & 56,1 & 65 \\
s12 & 52 & 177 & 50,7 & 65 \\
s13 & 52 & 146 & 42,6 & 90 \\
s14 & 52 & 100 & 38,8 & 90 \\
s15 & 52 & 100 & 33,0 & 90 \\
s16 & 52 & 83 & 35,1 & 90 \\
s17 & 52 & 73 & 40,5 & 90 \\
s18 & 52 & 110 & 49,5 & 90 \\
s19 & 52 & 162 & 59,9 & 90 \\
s20 & 52 & 143 & 67,5 & 90 \\
s21 & 52 & 117 & 61,4 & 90 \\
& & & &
\end{tabular}




\begin{tabular}{lllll} 
s22 & 52 & 84 & 48,5 & 90 \\
s23 & 52 & 94 & 39,9 & 90 \\
s24 & 52 & 87 & 31,5 & 90 \\
\hline
\end{tabular}

Table 8 Pre-agreed load curve

\begin{tabular}{|c|c|}
\hline $\begin{array}{c}\text { Time } \\
\text { interval }\end{array}$ & $\begin{array}{l}\text { Pre-agreed load } \\
\text { curve [MWh] }\end{array}$ \\
\hline s1 & 170,00 \\
\hline s2 & 146,17 \\
\hline s3 & 170,87 \\
\hline s4 & 153,57 \\
\hline s5 & 156,03 \\
\hline s6 & 182,20 \\
\hline s7 & 162,77 \\
\hline s8 & 155,77 \\
\hline s9 & 183,37 \\
\hline s10 & 157,70 \\
\hline s11 & 157,90 \\
\hline s12 & 182,33 \\
\hline s13 & 156,47 \\
\hline s14 & 174,33 \\
\hline s15 & 169,12 \\
\hline s16 & 99,90 \\
\hline s17 & 18,15 \\
\hline s18 & 15,73 \\
\hline s19 & 10,32 \\
\hline s20 & 7,00 \\
\hline s21 & 1,98 \\
\hline s22 & 0 \\
\hline s23 & 0 \\
\hline s24 & 0 \\
\hline
\end{tabular}

We assume that the income from selling back electricity to the grid is also time sensitive and is equal to $75 \%$ of the cost of day-ahead market in the same time slot. The cost of onsite generation and other related parameters are shown in Table 9. The allowed range for over- and under-consumption and the corresponding penalties can be found in Table 10, together with other load-deviation problem related parameters.

Table 9 Onsite generation parameters

\begin{tabular}{ll}
\hline Cost of onsite generation [€/MWh] & 61 \\
Minimum run time [h] & 3 \\
Minimum down time [h] & 3 \\
Start-up cost [€] & 1000 \\
Reduced production rate due to start-up & $20 \%$ \\
\hline
\end{tabular}

Table 10 Load deviation problem parameters

\begin{tabular}{ll}
\hline Over-conumption penalty [€/MWh] & 100
\end{tabular}

Under-consumption penalty [€/MWh] 80 


\subsection{Numerical results obtained with the monolithic models}

Numerical tests have been performed on a 4-core Intel Xeon 2,53GHz with 16GB of RAM using GAMS/CPLEX 23.7.3. The monolithic formulations shown in Section 4 and Appendix 1 were tested on the same instances with identical process assumptions and input data from Section 5.2. Table 11 gives the overview of the test cases involving different numbers of products to be scheduled, different time horizons, and the electricity prices considered.

Table 11 Test case description

\begin{tabular}{cccc}
\hline Scenario & Horizon & Products & Electricity sources and sinks \\
\hline 1 & $24 \mathrm{~h}$ & 20 & all possible, day-ahead with high prices \\
2 & $24 \mathrm{~h}$ & 20 & all possible, day-ahead with low prices \\
3 & $24 \mathrm{~h}$ & 16 & all possible, day-ahead with high prices \\
4 & $18 \mathrm{~h}$ & 12 & all possible, day-ahead with high prices \\
\hline Name & & Model type \\
\hline NM & monolithic six binaries model (Nolde and Morari 2010, Hadera and Harjunkoski 2013) \\
HM & monolithic event binary model & \\
\hline
\end{tabular}

The two monolithic model strategies are compared for different problem instances (scenarios) with computational limitations of 600s and 3600s. The resulting computational statistics are shown in Table 12. The MIP solution quality is described by the value of the total weighted objective function value.

Table 12 Numerical results of monolithic models

\begin{tabular}{rrrrrrrr}
\hline & \multicolumn{7}{c}{ Model statistics } \\
\hline Scenario & $\begin{array}{c}\text { Binary } \\
\text { vars }\end{array}$ & Total vars & Equations & $\begin{array}{c}\text { MIP } \\
\text { solution } \\
600 s\end{array}$ & $\begin{array}{c}\text { Relative gap } \\
600 \mathrm{~s}\end{array}$ & $\begin{array}{c}\text { MIP } \\
\text { solution } \\
3600 \text { s }\end{array}$ & $\begin{array}{c}\text { Relative gap } \\
3600 \mathrm{~s}\end{array}$ \\
\hline NM1 & 13017 & 29508 & 98095 & 313128 & $43,78 \%$ & 290708 & $38,97 \%$ \\
HM1 & 4065 & 29508 & 102335 & 247838 & $29,30 \%$ & 241136 & $26,80 \%$ \\
NM2 & 13017 & 29508 & 98095 & 223887 & $32,30 \%$ & 222167 & $31,20 \%$ \\
HM2 & 4065 & 29508 & 102335 & 200038 & $24,90 \%$ & 180023 & $16,10 \%$ \\
NM3 & 10181 & 23428 & 77136 & 234643 & $31,53 \%$ & 221454 & $27,20 \%$ \\
HM3 & 3229 & 23428 & 80528 & 204173 & $22,50 \%$ & 180965 & $12,10 \%$ \\
NM4 & 5723 & 13348 & 43541 & 197543 & $7,00 \%$ & 196886 & $6,16 \%$ \\
HM4 & 1771 & 13348 & 45509 & 194565 & $6,44 \%$ & 194361 & $6,20 \%$ \\
\hline
\end{tabular}

The results show that for most of the problem instances the new event binaries models (HM1-4) performs better than the literature model (NM1-4), especially for larger problems. This statement holds true for the short computational time limit (600s) where the differences of the relative gaps between the solutions is usually around 4-11\% (scenario 1-3). For longer solution times the improvement obtained with the binaries model is even more pronounced, around $12-15 \%$. Only for the smallest instance which is much smaller than the industrially relevant problems, with a scheduling horizon of $18 \mathrm{~h}$ and 12 products (scenario 4), the literature model had a performance comparable to the proposed one. 
In Table 13 the economic assessment of the resulting solutions is shown, for the computation time limit of 600s. The purchase strategy obtained from the solutions of the two monolithic models differs. However, in some cases for some contracts both models seem to be choosing the same levels of the flows in the network. For example, both models recognize that it is preferable to generate electricity from the power plant and to buy from TOU market when the day-ahead prices are high as in the case of scenarios 1,3 and 4 .

Table 14 gives the economic results for the test runs under the computational limitation of 3600s. When discussing the economic results it is important to note that the final objective function consists of three components, net electricity cost, penalties for load deviation and lead times (summation of task start times with the weight factor $c=1$ ). Therefore, the solver can find two very similar solutions in terms of the objective function values. However, the distribution of the costs among the elements of the objective function might differ. In Scenario 4 for 600s computation time the objective value for both model types is similar, but the six binaries model solution chooses to decrease the lead times at the expense of higher deviation penalties cost, while the event binaries model follows better the preagreed load curve causing the lead times to increase.

Table 13 Economic assessment results of the monolithic models - 600s computation time limit

\begin{tabular}{rrrrrrrr}
\hline \multicolumn{7}{c}{ Economic assessment -600 s } \\
\hline Scenario & $\begin{array}{c}\text { Lead } \\
\text { times } \\
{[\mathrm{min}]}\end{array}$ & $\begin{array}{c}\text { Net } \\
\text { electricity } \\
\text { cost [€] }\end{array}$ & $\begin{array}{c}\text { Electricity } \\
\text { purchase } \\
{[€]}\end{array}$ & $\begin{array}{c}\text { Deviation } \\
\text { penalties [€] }\end{array}$ & $\begin{array}{c}\text { Day-ahead } \\
\text { market } \\
\text { [MWh] }\end{array}$ & $\begin{array}{r}\text { TOU } \\
\text { [MWh] }\end{array}$ & $\begin{array}{c}\text { Onsite } \\
\text { generation } \\
\text { [MWh] }\end{array}$ \\
\hline NM1 & 60784 & 149832 & 161098 & 102512 & 172,55 & 1471,825 & 912 \\
HM1 & 51990 & 133972 & 151905 & 61876 & 173,49 & 1421,44 & 952 \\
NM2 & 61210 & 119440 & 98505 & 43236 & 1608,917 & 4 & 352 \\
HM2 & 53946 & 120989 & 98592 & 25103 & 1514,51 & 95,78 & 432 \\
NM3 & 43598 & 96266 & 134759 & 94780 & 77,207 & 1266,043 & 952 \\
HM3 & 36152 & 72846 & 140130 & 95175 & 142,48 & 1318,80 & 952 \\
NM4 & 22962 & 71410 & 107332 & 103171 & 0 & 1065,425 & 712 \\
HM4 & 24427 & 83226 & 103080 & 86912 & 0 & 1000 & 712 \\
\hline
\end{tabular}

Table 14 Economic assessment results of the monolithic models - 3600s computation time limit

\begin{tabular}{lcccrrrr}
\hline \multicolumn{7}{c}{ Economic assessment - 3600s } \\
\hline Scenario & $\begin{array}{c}\text { Lead } \\
\text { times } \\
{[\mathrm{min}]}\end{array}$ & $\begin{array}{c}\text { Net } \\
\text { electricity } \\
\text { cost [€] }\end{array}$ & $\begin{array}{c}\text { Electricity } \\
\text { purchase } \\
{[€]}\end{array}$ & $\begin{array}{c}\text { Deviation } \\
\text { penalties [€] }\end{array}$ & $\begin{array}{c}\text { Day-ahead } \\
\text { market } \\
\text { [MWh] }\end{array}$ & $\begin{array}{c}\text { TOU } \\
\text { [MWh] }\end{array}$ & $\begin{array}{c}\text { Onsite } \\
\text { generation } \\
\text { [MWh] }\end{array}$ \\
\hline NM1 & 58763 & 146373 & 162528 & 85572 & 203,72 & 1466,61 & 952 \\
HM1 & 50796 & 142452 & 14396 & 47888 & 234,22 & 1349,66 & 952 \\
NM2 & 60282 & 116872 & 98942 & 45014 & 1649,35 & 16,83 & 312 \\
HM2 & 51598 & 115937 & 98229 & 12489 & 1635,81 & 56,11 & 352 \\
NM3 & 60282 & 100104 & 127986 & 79081 & 110,87 & 1164,93 & 952 \\
HM3 & 35396 & 94723 & 128534 & 50846 & 46,66 & 1256,92 & 952 \\
NM4 & 23083 & 73788 & 105639 & 99956 & 0,00 & 1039,37 & 712 \\
HM4 & 24657 & 84528 & 103080 & 85177 & 0,00 & 1000 & 712 \\
\hline
\end{tabular}

The new model requires fewer binaries, while the total number of variables remains the same. The number of equations increases in the event binaries model. Unfortunately, the model is still not able to 
cope with the size of the industrial problem. Therefore, in the next sections we propose a heuristic strategy for the solution of the integrated scheduling and energy cost optimization problem.

\section{Bi-level heuristic}

When trying to solve an instance of the problem with significant flexibility in the process, i.e. when the optimization is free to assign and to sequence all products, the computational performance of the monolithic models from Section 4 is not sufficient. This is mainly due to large number of difficult to solve binary variables in the scheduling formulation and to the loose Big-M constraints, which is specific for precedence-based continuous-time models. To overcome the computational limitations we developed a heuristic decomposition strategy.

For large scale scheduling problems decomposition techniques have long been recognized as possible solution approaches. Starting from fundamental studies by Benders (1962) and Dantzig (1963) with row and column generation approaches, strategies have been developed for solving problems in an iterative fashion that could not be solved using a monolithic formulation. Decomposition approaches can be categorized into approaches that can be shown to converge to the true optimum (even if convergence may be slow) and approaches that discard a part of the solution space so that optimality cannot be guaranteed (decomposition heuristics). Wu and Ierapetritou (2003) presented a number of different heuristic decomposition approaches for scheduling problems. For example, one may use time decomposition where the long time horizon is divided into several smaller sub-periods with resulting sub-problems. Another important class of approaches make use of Lagrangean decomposition to relax the original problem into a problem that is easier to solve, systematically providing a lower bound for the solution. For problems with a clear separation of planning level decisions and scheduling level decisions these can be represented in a bi-level setup where first in upper level the planning variables are determined and then fixed to solve the more detailed lower level scheduling problem. This scheme was used for example by Bassett et al. (1996). Similarly in another example, Erdirik-Dogan and Grossmann (2008) use the bi-level concept for continuous multiproduct plants first solving an aggregate model to obtain an upper bound for the profit and then solving a scheduling problem to obtain a lower bound. Xu et al. (2012) developed a bi-level decomposition scheme for the coordination of a Melt Shop process with Hot Rolling section of a stainless steel plant. In this paper, we also employ a bi-level scheme, where the solution procedure consists of two problems that are solved in an iterative manner, as shown in Figure 8. 


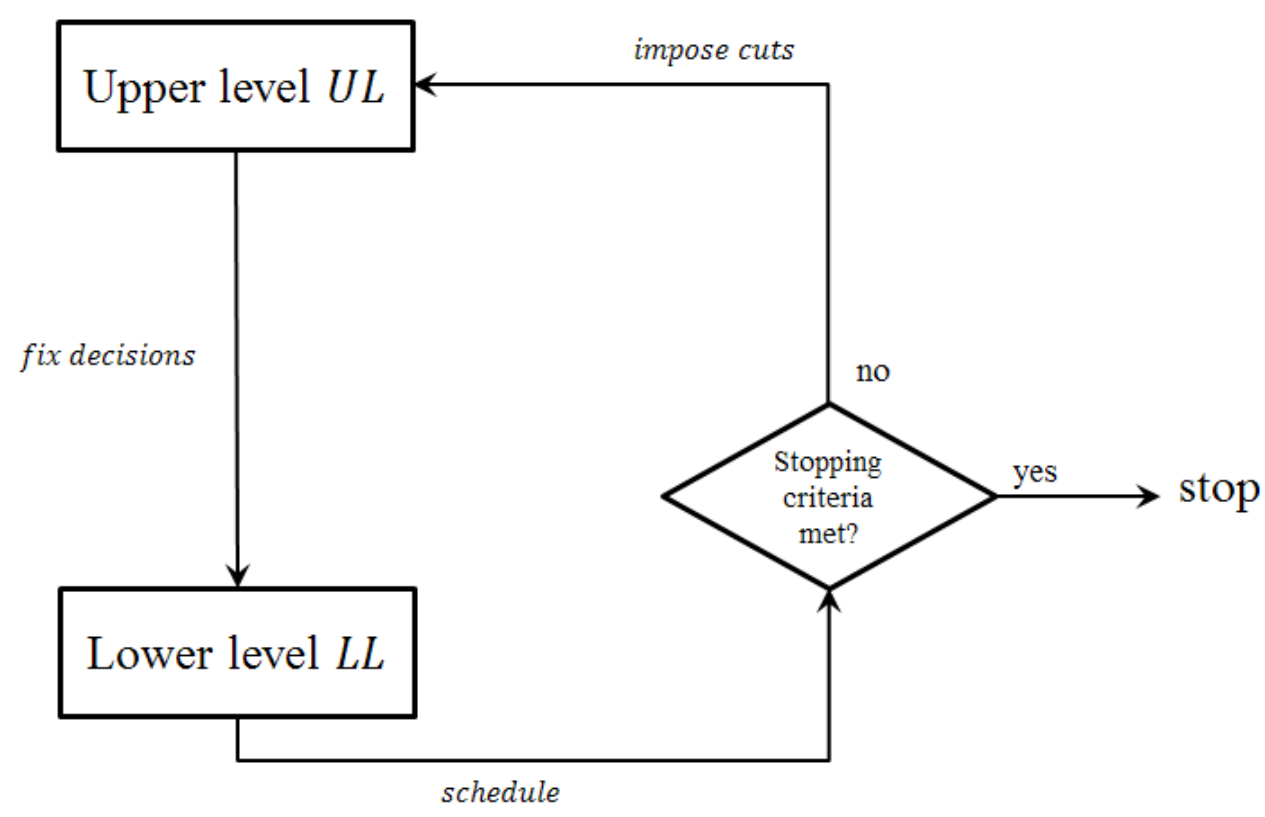

Figure 8 General idea of bi-level heuristic approach

First, an aggregate model (upper level $U L$ ) that approximates the original monolithic model is solved in order to obtain feasible values of some binary decisions. These binary decisions are passed to the full model (lower level $L L$ ) with a restriction to keep some of the variables fixed, optimizing some other continuous and binary variables, in our case the starting times and the event binaries. The full model should provide a feasible schedule and an objective function value which represents an upper bound of the optimal value. A new iteration of the algorithm starts by solving the upper problem again, with some new restrictions in the form of integer cuts that exclude previous solutions of the full model. The search space can be reduced based on the knowledge about the optimal solution provided from $L L$. In our particular case, since for the full problem with some of the decision variables fixed a feasible solution was obtained, at least the combination of the binaries of that solution can be removed from $U L$ so that new values of these binaries are generated by the upper level model and the new solution is again refined by the lower level model. The algorithm iterates until stopping criterion is met, e.g. until a time limit is exceeded. For the following sections, additional notation specific for the decomposition approach is given in Table 15.

Table 15 Model notation for the heuristic

\begin{tabular}{|c|c|}
\hline \multicolumn{2}{|l|}{ Sets: } \\
\hline$D Y_{0}^{r}, D Y_{1}^{r}, D X_{0}^{r}, D X_{1}^{r}, D V_{0}^{r}, D V_{1}^{r}$ & $\begin{array}{l}\text { dynamic sets used in bi-level heuristic for false and true decision of } \\
\text { the respective binaries }\end{array}$ \\
\hline \multicolumn{2}{|r|}{ 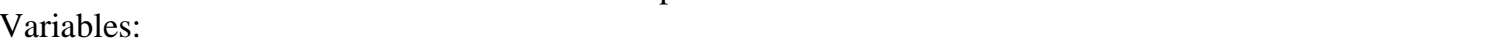 } \\
\hline$X_{m, p}^{U L 1}, X_{m, p}^{L L}, X_{m, p}^{U L 2}$ & $\begin{array}{l}\text { binary variable in respective models UL1, LL and UL2, true when } \\
\text { heat } p \text { is assigned for processing on equipment } m\end{array}$ \\
\hline$V_{s t, p, p^{\prime}}^{U L 1}, V_{s t, p, p^{\prime}}^{L L}, V_{s t, p, p \prime}^{U L 2}$ & $\begin{array}{l}\text { binary variable in respective models UL1, LL and UL2, true when } \\
\text { heat p' is processed after heat p on stage st }\end{array}$ \\
\hline$Y_{p, s t, s}^{s U L 1}, Y_{p, s t, s}^{s L L}, Y_{p, s t, s}^{s U L 2}$ & $\begin{array}{l}\text { binary variable in respective models UL1, LL and UL2, true when } \\
\text { heat p starts on stage st in the slot s }\end{array}$ \\
\hline \multicolumn{2}{|l|}{ Parameters: } \\
\hline$t_{m, m^{\prime}}^{\min U L 1}, t_{m, m^{\prime}}^{\min L L}$ & $\begin{array}{l}\text { minimum transport time from equipment } \mathrm{m} \text { to } \mathrm{m} \text { ' in respective } \\
\text { models UL1 and LL }\end{array}$ \\
\hline$t_{p, s t}^{\max U L 1}, t_{p, s t}^{\max L L}$ & $\begin{array}{l}\text { maximum hold-up time after stage st in respective models UL1 and } \\
\text { LL }\end{array}$ \\
\hline RHS & sum of binary variables \\
\hline$\alpha$ & number of neighboring slots to be evaluated \\
\hline
\end{tabular}




\begin{tabular}{ll}
\hline$\beta$ & desired optimality gap \\
$r$ & iteration number \\
\hline
\end{tabular}

\subsection{Upper level problem}

The upper level problem $U L$ consist of solving two models $U L 1$ and $U L 2$ as shown in Figure 9. The $U L 1$ is a simplified model of the original problem and it is computed to obtain a valuable guess of some binary decisions, while the $U L 2$ is a pre-computation step for the $U L 1$ starting from the second iteration as explained later. The algorithm starts with solving $U L 1$, which is constructed in such way that it represents the full monolithic problem as closely as possible, while at the same time reducing the size of the MILP. In the first iteration, $U L 1$ is a relaxation of the full problem. The main component of the objective function value is the electricity-related cost. It depends directly on the load pattern that results from the processing of the tasks. In the stainless-steel production process investigated in the case study, the EAF stage consumes about $88 \%$ of the total electricity needed to deliver one product. Therefore potential changes in the assignments, sequences or especially the timing of different products on that stage will have a significant impact on the final consumption pattern. Therefore, the energy-intense melting task is included in the upper level problem. A rough approximation of the lower level problem can be generated by simply scheduling the EAF stage alone, maintaining all energy-related constraints. However, the tasks on the first stage cannot be timed arbitrarily and must be sequenced according to the special continuous-casting constraints on the CC stage. For example, two subsequently casted products should be processed within a reasonable time interval in the EAF stage in order to ensure the proper delivery of the heats to continuous-casting, while at the same time satisfying all transfer and waiting time constraints between the stages.

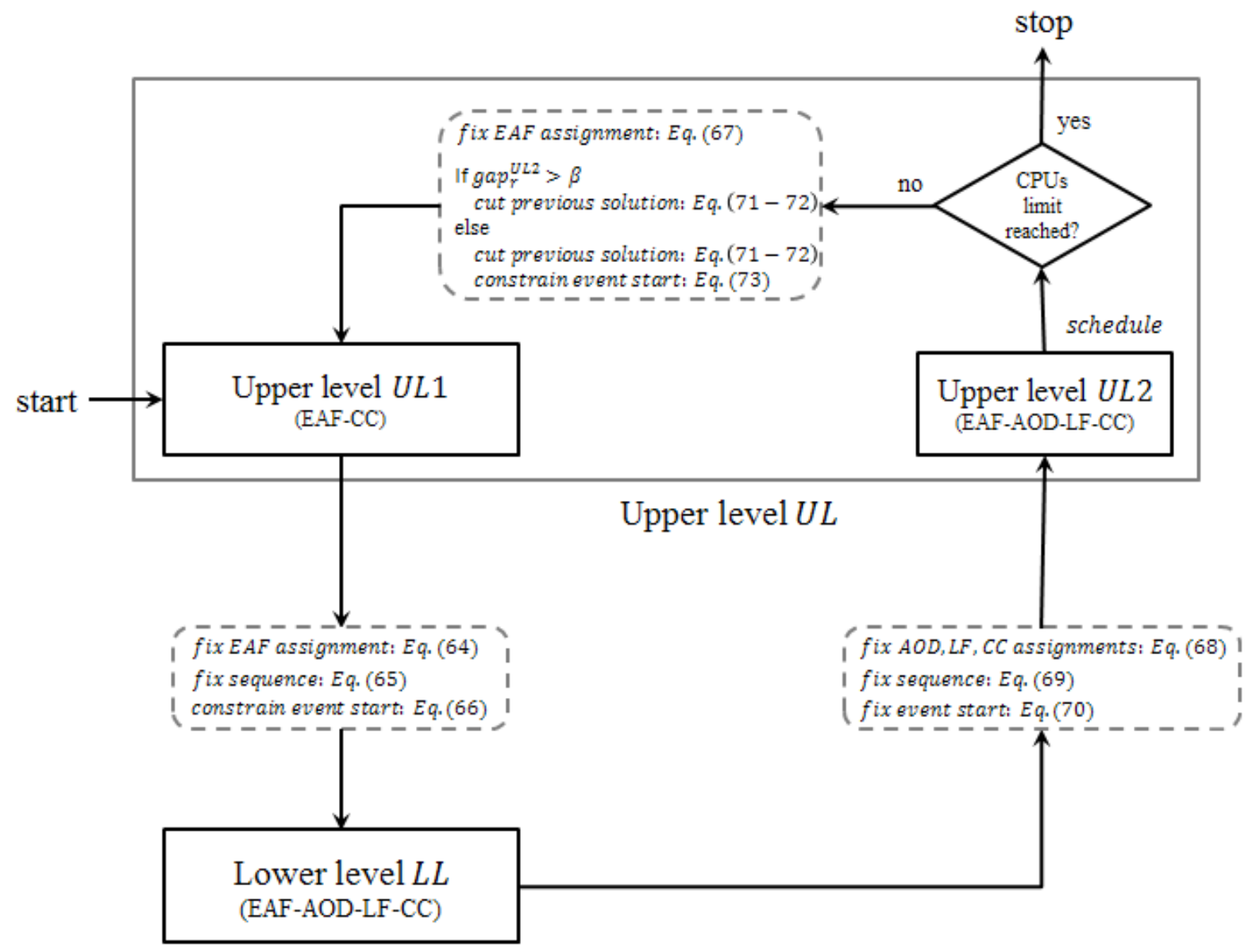


Figure 9 Bi-level heuristic algorithm

Since the EAF and CC stages together account for around 95\% of the total Melt Shop electricity consumption, scheduling of these two stages alone should produce a good guess of the values of the variables related to the EAF and the $\mathrm{CC}$ of the full problem. If the last production stage is considered together with the EAF, the casting constraints are not violated and the remaining stages of AOD and LF can be scheduled on the lower level. In order to ensure feasibility of the lower model concerning decisions for these two stages, the upper level problem needs to account for the range of possible delays between processing on the EAF and on the CC stage. The equations of the $U L 1$ problem are the same as in the corresponding monolithic model (and in lower level problem), apart from removing elements and cuts, as follows:

$$
\min ^{U L 1}(E q .61)
$$

s.t.:

Eq. $(1-16) \quad$ Scheduling model equations with new sets $M$ and $S T$ (Section 4.2)

Eq. (17 - 46) Energy-awareness extension with new sets $M$ and $S T$ (Section 4.3.1)

Eq. (47 - 57) Electricity sources optimization (Section 4.4.1)

Eq. (58 - 60) Load deviation problem (Section 4.4.2)

Cuts New constraints for other iterations than the initial one (Section 6.4)

The equipment AOD and LF are eliminated from the equipment set $M$. The stages st 2 and st 3 are eliminated from the set of production stages $S T$. Therefore, new values of the minimum transport times and maximum hold-up times between EAF and CC stage in the upper level problem need to be calculated based on the parameters of the full model as shown in Figure 10.

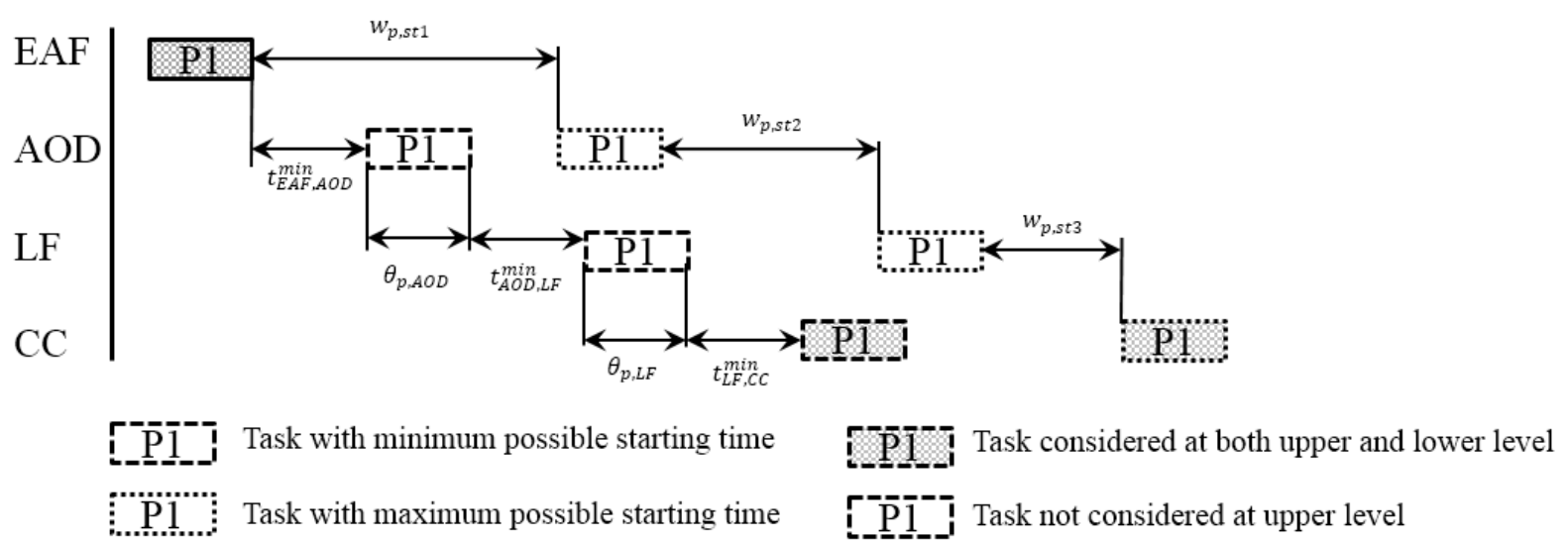

Figure 10 Transportation and waiting time between EAF and CC stage in the upper level UL1 problem

The new $t_{p, s t}^{\max U L 1}$ and $t_{m, m^{\prime}, C C}^{\min U L}$ replace the original $t_{p, s t}^{\max }$ and $t_{m, m^{\prime}}^{\min }$ from the monolithic model. The maximum hold-up time corresponds in the full model to the maximum time after which a heat can be processed on CC after finished on EAF as in Equation (62) below. 


$$
\begin{aligned}
& t_{p^{\prime}, s t 1}^{\max U L 1}= \\
& \max _{\mathrm{p} \in \mathrm{P}}\left\{t_{p, s t 1}^{\max }\right\}+\max _{\mathrm{p} \in \mathrm{P}}\left\{\tau_{p, A O D 1}, \tau_{p, A O D 2}\right\}+\max _{\mathrm{p} \in \mathrm{P}}\left\{t_{p, s t 2}^{\max }\right\}+\max _{\mathrm{p} \in \mathrm{P}}\left\{\theta_{p, L F 1}, \theta_{p, L F 2}\right\}+ \\
& \max _{\mathrm{p} \in \mathrm{P}}\left\{w_{p, s t 3}^{\max }\right\} \\
& \forall p^{\prime} \in P
\end{aligned}
$$

Similarly, the minimum transportation time between EAF and CC corresponds to the minimum possible time between these two in the full problem as in Equation (63).

$$
\begin{aligned}
& t_{E A F, C C}^{\min U L 1}=\min _{\mathrm{m} \in \mathrm{SM}(\mathrm{EAF}, \mathrm{m})}\left\{t_{m, m^{\prime}}^{\min }\right\}+\min _{\mathrm{p} \in \mathrm{P}}\left\{\theta_{p, A O D 1}, \theta_{p, A O D 2}\right\}+\min _{\mathrm{m} \in \mathrm{SM}(\mathrm{AOD}, m)}\left\{t_{m, m^{\prime}}^{\min }\right\}+ \\
& m^{\prime} \in \operatorname{SM}\left(\mathrm{LF}, m^{\prime}\right) \\
& \min _{\mathrm{p} \in \mathrm{P}}\left\{\theta_{p, L F 1}, \theta_{p, L F 2}\right\}+\min _{\operatorname{m} \in \operatorname{SM}(L F, m)}\left\{t_{m, m \prime}^{\min }\right\} \\
& m^{\prime} \in \operatorname{SM}\left(\mathrm{CC}, m^{\prime}\right)
\end{aligned}
$$

In the upper level model $U L 1$, the EAF stage is the first stage, followed by the CC which is the second and last production stage. Another modification of the input data of the upper level problem concerns the pre-agreed load curve. For the original full problem, the agreed curve is calculated based on a predefined schedule. For the same schedule, it is possible to eliminate the AOD and LF stages to obtain a load curve for the other two stages.

The second model $U L 2$ of the upper level is solved after the lower level $L L$ problem as shown in Figure 9. From the latter, most binary decisions are fixed and transferred to $U L 2$, which essentially is the same problem as $L L$ discussed in the next section. However, within $U L 2$ the only binary decision to be determined by optimization is to find better assignments of heats to EAFs in order to precompute new assignment decisions on EAFs for the next iteration of UL1. In this way the search space of the approximate model $U L 1$ is reduced and it no longer is a relaxation of original problem in the later iterations, which might prevent finding the optimal solution. However, it turned out to speed up the computational time significantly.

\subsection{Lower level problem}

The constraints and sets of the lower level $L L$ problem are not changed compared to the monolithic problem. However, the lower problem is solved with some fixed decisions which improves its computational performance, as discussed in details in the next Section. The model $L L$ serves as an evaluation model for the decisions that were determined by the upper level $U L 1$. After fixing some decisions, as described in the next section, $L L$ is solved with a limitation on the solution time to avoid spending too much time in closing a small optimality gap.

\subsection{Information exchange between the levels}

Since the EAF stage is the most power intensive one, the decisions taken with regard to the assignment $X_{p, m}$ to machines of the melting stage are fixed for the $L L$ problem as in Equation (64), which helps to speed up the solving time. Further, for the same reason another variable is fixed, the sequence $V_{s t, p, p^{\prime}}$ on the casting stage as in Equation (65). In contrast to the process assumptions where the sequence in the particular caster is known a priori only if only one caster can be used as described in Section 5, we fix the sequence relation of the products between the two casters here which is a degree of freedom of the monolithic problem.

$$
\begin{array}{ll}
X_{m, p}^{L L}=X_{m, p}^{U L 1} & \forall p \in P, m \in E A F \\
V_{s t, p, p^{\prime}}^{L L}=V_{s t, p, p \prime}^{U L 1} & \forall p, p^{\prime} \in P, p \neq p^{\prime}, s t=|S T|
\end{array}
$$

Since the upper problem should provide a good approximation of the full problem, it would be beneficial to use also the energy-related information obtained from it for fixing some decisions in the 
lower level problem. A natural choice is the event binaries. However, since it is expected that these have a large impact on the value of the objective function, the kind of fixing needs to be carefully chosen. The fixing should still allow for giving flexibility to the model, and at the same time reduce the computational time of the full problem. After experimenting with different options, we developed a fixing decision that if the upper level problem is solved close to optimality (i.e. the gap is lower than $\beta=2 \%$ ) the variables of event binary start of the lower level problem $Y_{p, s t, s}^{s L L}$ should be true within a neighborhood of the slots for which the binary holds true in the $U L 1$ solution as shown in Equation (4) where $s^{*}$ denotes the time slot when the event binary starts to hold true in the $U L 1$ solution.

$\sum_{s=s^{*}-\alpha}^{s^{*}+\alpha} Y_{p, s t, s}^{s L L}=1$, where $s^{*}: Y_{p, s t, s^{*}}^{s U L 1}=1 \quad \forall p \in P, s t \in S T$

If the upper level problem determined that the start of a product should occur in the $n-t h$ time slot, then the start of that product in the $L L$ solution should occur in one of the time slots within ( $n-$ $\alpha ; n+\alpha)$. For the particular case, we choose $\alpha$ to define a neighborhood of 3 slots, which is a wide range of 7 hours in total. Since the decision of the event start binary has a direct impact on the event finish binary, there is no need for further fixing of the latter.

With the above exchange of information between models $U P 1$ and $L L$, the most important degrees of freedom in the lower level problem are the timing of EAFs (but also all the other units) while keeping the sequence determined by the upper level.

In order to update the $U L 1$ problem with new assignment decisions on EAFs (Equation 67), the $U L 2$ problem is solved with fixed decisions of the other binaries, as shown in Equations (68-70).

$X_{m, p}^{U L 1}=X_{m, p}^{U L 2} \quad \forall p \in P, m \in E A F$

$X_{m, p}^{U L 2}=X_{m, p}^{L L} \quad \forall p \in P, m \in M \backslash E A F$

$V_{s t, p, p,}^{U L 2}=V_{s t, p, p}^{L L} \quad \forall p, p^{\prime} \in P, p \neq p^{\prime}$, st $\in S T$

$Y_{p, s t, s}^{s U L 2}=Y_{p, s t, s}^{s L L} \quad \forall p \in P, s t \in S T, s \in S$

The UP2 model has very few degrees of freedom since it can only change the binaries related to the EAF assignment. In contrast, in $U P 1$ is the one where many important decisions are made since this model finds the timing and sequence of products on the most important units, especially on the EAF. The latter are then fixed at the lower level.

\subsection{Cuts and stopping criteria}

In the proposed approach, cuts imposed in each iteration are related to the scheduling decisions $\left(X_{m, p}, V_{p, p^{\prime}, s t}\right)$ and the energy-awareness $\left(Y_{p, s t, s}^{s}\right)$. Of course the latter ones are strongly related to the former since it is the timing of a task start which links both. In the case when $L L$ is not proven to have a desired level of optimality, we can suspect that the decisions obtained from it might not be good enough to later cut off the neighborhood of the obtained solution of event binary start variables from the solution space of $U L 1$. Therefore, if for a particular iteration the desired optimality level is not obtained in the $L L$ problem, the cut for the $U L 1$ involves only removing a particular solution of $L L$, which means a particular combination of the binaries $X_{m, p}, V_{p, p^{\prime}, s t}, Y_{p, s t, s}^{S}$ obtained in $L L$ as there is no need of evaluating that solution again in new iteration in the upper level problem. The cut is achieved by the constraints shown in Equations (71-72), similar to those reported (Balas and Jeroslow 1972) and successfully used in the literature (Iyer and Grossmann 1998) for the elimination of existing binary solutions. In case where $L L$ is solved to optimality we can enforce a stronger cut, removing also 
the neighborhood of the event binary start as shown in Equation (73), following the fixing in Equation (66) coming from the $U L 1$.

$$
\begin{aligned}
& R H S=\sum_{p \in P, s \in S} Y_{p, s t 1, S}^{S U L 1}+\sum_{m \in M, p \in P} X_{m, p}^{U L 1}+\sum_{p, p^{\prime} \in P, p \neq p^{\prime}} V_{s t 4, p, p^{\prime}}^{U L 1} \\
& \sum_{(p, s) \in D Y_{1}^{r}} Y_{p, s t 1, s}^{s U L 1}-\sum_{(p, s) \in D Y_{0}^{r}} Y_{p, s t 1, s}^{s U L 1}+\sum_{(m, p) \in D X_{1}^{r}} X_{m, p}^{U L 1}-\sum_{(m, p) \in D X_{0}^{r}} X_{m, p}^{U L 1}+\sum_{\left(p, p^{\prime}\right) \in D V_{1}^{r}} V_{s t 4, p, p^{\prime}}^{U L 1}- \\
& \sum_{\left(p, p^{\prime}\right) \in D V_{0}^{r} V_{s t}^{U L, p, p^{\prime}}} \leq R H S-1 \\
& D Y_{0}^{r}=\left\{(p, s) \mid Y_{r, p, s t 1, s}^{s U L 1}=0\right\} D Y_{1}^{r}=\left\{(p, s) \mid Y_{r, p, s t 1, s}^{s U L 1}=1\right\}, \\
& D X_{0}^{r}=\left\{(m, p) \mid X_{r, m, p}^{U L 1}=0\right\} D X_{1}^{r}=\left\{(m, p) \mid X_{r, m, p}^{U L 1}=1\right\} \text {, } \\
& D V_{0}^{r}=\left\{\left(p, p^{\prime}\right) \mid V_{s t 4, p, p^{\prime}}^{U L 1}=0\right\} D V_{1}^{r}=\left\{\left(p, p^{\prime}\right) \mid V_{s t 4, p, p^{\prime}}^{U L 1}=1\right\}, p \neq p^{\prime} \\
& \sum_{\left(p, s^{\prime}\right) \in D Y_{1}^{r}} Y_{p, s t 1, s^{\prime}+\gamma}^{s U L 1}-\sum_{\left(p, s^{\prime}\right) \in D Y_{0}^{r}} Y_{p, s t 1, s^{\prime}+\gamma}^{s U L 1}+\sum_{(m, p) \in D X_{1}^{r}} X_{m, p}^{U L 1}-\sum_{(m, p) \in D X_{0}^{r}} X_{m, p}^{U L 1}+ \\
& \sum_{\left(p, p^{\prime}\right) \in D V_{1}^{r}} V_{s t 4, p, p^{\prime}}^{U L 1}-\sum_{\left(p, p^{\prime}\right) \in D V_{0}^{r}} V_{s t 4, p, p^{\prime}}^{U L 1} \leq R H S-1 \quad \forall \gamma \in(-\alpha ;+\alpha), \alpha=3 \\
& D Y_{0}^{r}=\left\{\left(p, s^{\prime}\right) \mid Y_{r, p, s t 1, s^{\prime}}^{s U L 1}=0\right\} D Y_{1}^{r}=\left\{\left(p, s^{\prime}\right) \mid Y_{r, p, s t 1, s^{\prime}}^{s U L 1}=1\right\}, \\
& D X_{0}^{r}=\left\{(m, p) \mid X_{r, m, p}^{U L 1}=0\right\} D X_{1}^{r}=\left\{(m, p) \mid X_{r, m, p}^{U L 1}=1\right\}, \\
& D V_{0}^{r}=\left\{\left(p, p^{\prime}\right) \mid V_{s t 4, p, p}^{U L 1}=0\right\} D V_{1}^{r}=\left\{\left(p, p^{\prime}\right) \mid V_{s t 4, p, p^{\prime}}^{U L 1}=1\right\}, p \neq p^{\prime}
\end{aligned}
$$

The algorithm performs the iterative steps as shown in Figure 9. The upper problem $U L 1$ is not a strict mathematical relaxation except of the first iteration, therefore we cannot use the objective function to systematically close the gap between the lower and the upper bounds, as it was the case for example in Iyer and Grossmann (1998). In the later iterations, $U L 1$ is not a relaxed problem of the monolithic model because it considers the assignment of the EAFs as fixed and as long as this fixing is not optimal the solution from the upper level problem is not a valid lower bound. The assignments coming from $U L 2$ to $U L 1$ are used to speed up the computation time of solving $U L 1$, which most of the times is not solvable to near-optimal solutions in short times, thus giving weak solution without the fixing. It is reasonable to use the fixing also because of its much lower importance on the objective function compared to the degrees of freedom that the $U L 1$ is handling, namely timing and sequencing.

Therefore, the solution of $U L 1$ does not provide an increasing lower bound. At the same time the lower level problem $L L$ and $U L 2$ provide upper bounds as a feasible solution of the monolithic problem is obtained - the latter is always at least as good as the one from $L L$. Since the proposed algorithm does not guarantee to converge to the optimal solution, the most reasonable stopping criterion for the iterative execution is the total time spent on computations or the desired number of iterations, which is acceptable for industrial practice as long as the algorithms yields good quality solutions in reasonable computation times.

\subsection{Application of the heuristic to the industrial case study}

We tested the bi-level heuristic on the same problem instances as the monolithic model. In the decomposition scheme, some modifications of the input data are needed, due to the elimination of the AOD and LF stages. The new maximum waiting times and minimum transportation times of the upper level $U L 1$ that were calculated using Equations (62-63) are shown in Table 16 and Table 17.

Table 16 Upper level $U L 1$ problem maximum waiting times

\begin{tabular}{ccc}
\hline & ST1 & ST2 \\
\cline { 2 - 3 } P1-P20 & 161 & 90 \\
\hline
\end{tabular}


Table 17 Upper level $U L 1$ problem minimum transportation times

\begin{tabular}{lcc}
\hline & CC1 & CC2 \\
\cline { 2 - 3 } EAF1 & 155 & 161 \\
EAF2 & 161 & 155 \\
\hline
\end{tabular}

The committed load curve for the upper level problem $U L 1$ is modified by considering the lower consumption due to omitting the AOD and LF stages as shown in Table 18.

Table 18 Pre-agreed load curve for the upper level $U L 1$ problem

\begin{tabular}{lr}
\hline $\begin{array}{c}\text { Time } \\
\text { interval }\end{array}$ & $\begin{array}{r}\text { Pre-agreed load } \\
\text { curve UL1 [MWh] }\end{array}$ \\
\hline s1 & 170,00 \\
s2 & 144,50 \\
s3 & 167,17 \\
s4 & 147,33 \\
s5 & 151,03 \\
s6 & 177,00 \\
s7 & 157,10 \\
s8 & 151,97 \\
s9 & 177,00 \\
s10 & 153,37 \\
s11 & 152,90 \\
s12 & 177,00 \\
s13 & 151,50 \\
s14 & 168,50 \\
s15 & 163,15 \\
s16 & 94,83 \\
s17 & 12,02 \\
s18 & 14,00 \\
s19 & 8,98 \\
s20 & 7,00 \\
s21 & 1,98 \\
s22 & 0 \\
s23 & 0 \\
s24 & 0 \\
\hline &
\end{tabular}

\subsubsection{Numerical results of the heuristic approach}

Since the heuristic approach does not guarantee to provide systematically a better upper bound with each iteration the best solution of $U L 2$ among all iterations is considered to be the bi-level algorithm's solution (scenarios H1-4). Therefore, $U L 2$ 's solution statistics are reported in Table 19 which shows that the approach is always able to find better quality solutions within the given time limit compared to the monolithic formulation. To assess the solution obtained from the heuristic (relative gap) we compared the solution with a best bound (LP relaxation reported by the solver) obtained from optimization runs of the monolithic model with a computation limit of $1 \mathrm{~h}$ and the heuristic solution being provided as the initial solution for the solver. To fairly compare the monolithic result, we also calculated new gaps for the solutions of the monolithic models HM1-4 previously reported in Table 12 for 600 s computation time. 
The heuristic decomposition always obtains better solutions than the monolithic solution, usually $4-9$ $\%$ different from best relaxation found. Only for the smallest problem instance Scenario 4 did the heuristic algorithm provide comparably good solutions without a significant improvement. The quality of these solutions is expected to be better, however it is difficult to find a best bound that would asses it. Very good results of the heuristic decomposition are achieved already in the first iteration. Often after up to 3-4 iterations the best solution is found. In Figure 11 the evolution of objective function values for all models in Scenario 1 is shown. It can be observed that the $U L 1$ values in each iteration of the algorithm are constant, even though due to the cuts each iteration finds different solution from all the previous ones. This is due to the fact that a slight change in the timing and assignment or sequence of products (while satisfying the cuts) is very likely to give the same objective value since there are many similar solutions in $U L 1$. However, when solving the more detailed $L L$ model the values are changing in each iteration in response to the different decisions taken in $U L 1$. For the same reason the objective function value of $L L$ can improve in further iterations since there are AOD and LF stages added as well as the new load deviation curve. Here it can be noted that the solution quality is not expected to improve significantly in further iterations as the bi-level solution method is based on the idea that the upper level should provide a very good rough schedule already in the first iteration. The objective function value of $U L 2$ always improves the solution from $L L$ slightly by finding a better assignment on EAF units. It should be also noted that for Scenario 2-4 the objective function value of $U L 1$ is lower than $L L$ and $U L 2$, however this is not true for Scenario 1. The reason that higher values might appear in approximated $U L 1$ is larger deviation penalties paid than in detailed $L L$ and $U L 2$. A general behavior of the algorithm very similar to the one shown in Figure 11 was observed for all of the investigated scenarios.

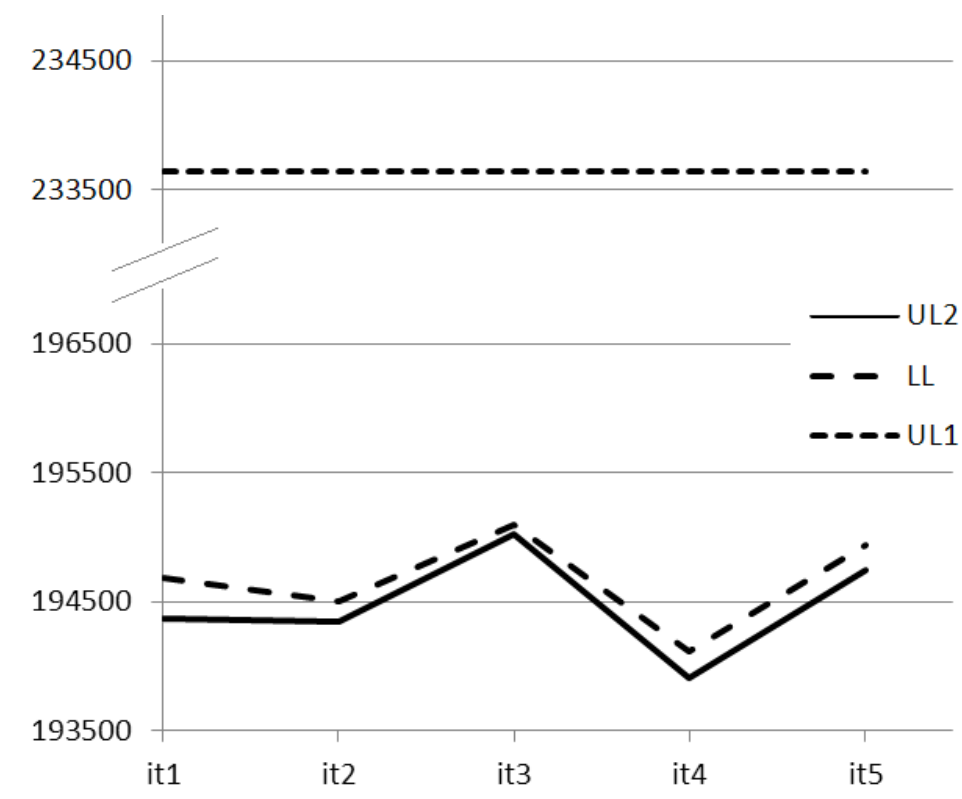

Figure 11 Objective function value change in each iteration for all models of Scenario 1 
Table 19 Numerical results for monolithic (HM) and bi-level heuristic (H) approaches - 600s computation limit

\begin{tabular}{|c|c|c|c|c|c|c|c|c|c|c|c|c|c|c|c|}
\hline \multicolumn{8}{|c|}{ Model statistics } & \multicolumn{8}{|c|}{ Economic assessment } \\
\hline Scenario & $\begin{array}{l}\text { Binary } \\
\text { vars } \\
\text { (UL2) }\end{array}$ & $\begin{array}{l}\text { Total } \\
\text { vars } \\
\text { (UL2) }\end{array}$ & $\begin{array}{l}\text { Equations } \\
\text { (UL2) }\end{array}$ & $\begin{array}{l}\text { MIP } \\
\text { solution } \\
\text { (UL2) }\end{array}$ & $\begin{array}{l}\text { MIP } \\
\text { solution } \\
\text { LL }\end{array}$ & $\begin{array}{l}\text { MIP } \\
\text { solution } \\
\text { UL1 }\end{array}$ & $\begin{array}{l}\text { Relative } \\
\text { gap }\end{array}$ & $\begin{array}{l}\text { Lead } \\
\text { times } \\
{[\mathrm{min}]}\end{array}$ & $\begin{array}{c}\text { Net } \\
\text { electricity } \\
\text { cost } \\
{[€]}\end{array}$ & $\begin{array}{l}\text { Electricity } \\
\text { purchase } \\
{[€]}\end{array}$ & $\begin{array}{c}\text { Deviation } \\
\text { penalties } \\
{[€]}\end{array}$ & $\begin{array}{c}\text { Day-ahead } \\
\text { market } \\
\text { [MWh] }\end{array}$ & $\begin{array}{l}\text { TOU } \\
{[\mathrm{MWh}]}\end{array}$ & $\begin{array}{c}\text { Onsite } \\
\text { generation } \\
\text { [MWh] }\end{array}$ & $\begin{array}{l}\text { No. of } \\
\text { iterations } \\
\text { (best) }\end{array}$ \\
\hline HM1 & 4065 & 29508 & 102335 & 247838 & & & $29,10 \%$ & 51990 & 133972 & 151905 & 61876 & 173,49 & 1421,44 & 952 & - \\
\hline H1 & 1458 & 29508 & 102335 & 193904 & 194118 & 233637 & $9,89 \%$ & 45847 & 147252 & 156934 & 806 & 177,484 & 1455,972 & 952 & $5(4)$ \\
\hline HM2 & 4065 & 29508 & 102335 & 200038 & & & $23,39 \%$ & 53946 & 120989 & 98592 & 25103 & 1514,51 & 95,78 & 432 & - \\
\hline H2 & 1458 & 29508 & 102335 & 165198 & 165267 & 161329 & $9,09 \%$ & 45459 & 119451 & 103985 & 288 & 1519,967 & 229,183 & 352 & $5(3)$ \\
\hline HM3 & 3229 & 23428 & 80528 & 204173 & & & $21,97 \%$ & 36152 & 72846 & 140130 & 95175 & 142,48 & 1318,80 & 952 & - \\
\hline H3 & 1276 & 23428 & 80528 & 174796 & 177664 & 165428 & $9,80 \%$ & 31248 & 95189 & 127981 & 48358 & 77,371 & 1244,879 & 952 & 3(3) \\
\hline HM4 & 1771 & 13348 & 45509 & 194565 & & & $4,00 \%$ & 24427 & 83226 & 103080 & 86912 & 0 & 1000 & 712 & - \\
\hline $\mathrm{H} 4$ & 724 & 13348 & 45509 & 194607 & 195586 & 186603 & $3,79 \%$ & 22450 & 79122 & 104481 & 93035 & 0 & 1029,895 & 712 & $5(4)$ \\
\hline
\end{tabular}




\section{Conclusions and remarks}

In this paper we have proposed a new strategy for embedding energy-awareness into a continuoustime scheduling approach which optimizes the production schedules of energy-intensive plants (Section 3) under consideration of to time-sensitive prices of electricity and load commitment penalties (Section 4). The proposed approach was compared to a model available in the literature. The numerical experiments (Section 5.3) show that the use of the new event binaries is more efficient. However, both monolithic models cannot be solved within the available computation times for largescale industrial problem instances. Therefore, we developed a bi-level decomposition-based heuristic (Section 6) to obtain good quality results in reasonable computation times.

The proposed solution scheme benefits from the exact timing of the tasks by the continuous-time scheduling representation. The model is able to capture complex price structures and to optimally determine the exact amount of electricity to be purchased and sold. The flexible part of the purchase optimization can be further extended by more complex dependencies between the contracts. The model might help assessing different price levels of negotiated contracts, as well as reducing the risk associated with volatile electricity markets. An important restriction is that the plant needs to make commitments on the amounts to be bought and sold on the day-ahead markets. Even more important factors are the disturbances and the technical capability to implement the optimized schedule.

To address further the limitations of the model concerning computational performance for large instances, a scheduling horizon of several days could be investigated with a rolling horizon approach. Decisions for longer time windows should be done with higher level short- and long-term planning solutions taking into account different factors than those considered by the scheduling level. Further work could also deal with improvements of the developed algorithm towards a more rigorous scheme.

\section{Acknowledgments}

We would like to acknowledge the Marie Curie FP7-ITN project "Energy savings from smart operation of electrical, process and mechanical equipment- ENERGY-SMARTOPS", Contract No: PITN-GA-2010-264940 for financial support. 


\section{References}

Ashok, S., 2006, Peak-load management in steel plants, App. Energy, 83, 5, 413-424

Balas, E., Jeroslow, R., 1972, Canonical cuts on the unit hypercube. SIAM Journal of Applied Mathematics 23 (1), 61-69

Bassett, M., Pekny, J. F., Reklaitis, G. V., 1996, Decomposition techniques for the solution of largescale scheduling problems, AIChE Journal, 42, 12, 3373-3387

Benders, J. F., 1962, Partitioning procedures for solving mixed-variables programming problems, Numer. Math. 4, 3, 238-252

BGFRS - Board of Governors of Federal Reserve System, 2013, Industrial Production and Capacity Utilization, http://www.federalreserve.gov/datadownload/Download.aspx?rel=G17\&series=0fc8d1a1edcda88d7d3 db1971fa6d4b8\&filetype=spreadsheetml\&label=include\&layout=seriescolumn\&from=01/01/1950\&to $=12 / 31 / 2013$, accessed 02.02.2014

Boukas, E. K., Haurie, A. and Soumis, F., 1990, Hierarchical approach to steel production scheduling under a global energy constraints, Annals of Operations Research, 26, 171-184

Castro, P., Grossmann, I. E., Veldhuizen, P., Esplin, D., 2014, Optimal maintenance scheduling of a gas engine power plant using generalized disjunctive programming. AIChE J., 60: 2083-2097. doi: 10.1002/aic.14412

Castro, P., Harjunkoski, I., Grossmann, I. E., 2009, New continuous-time scheduling formulation for continuous plants under variable electricity cost, Industrial and Engineering Chemistry Research, 48, $14,6701-6714$

Castro, P., Harjunkoski, I., Grossmann, I. E., 2011, Optimal scheduling of continuous plants with energy constraints, Computers and Chemical Engineering, 35, 2, 372-387

Castro, P., Sun, L., Harjunkoski, I., 2013, Resource-Task Network Formulations for Industrial Demand Side Management of a Steel Plant, Ind. Eng. Chem. Res., 52, 36, 13046-13058

CRA (Charles River Associates), 2005, Primer on demand-side management, The World Bank

Dantzig, G.B., 1963, Linear Programming and Extensions, Princeton University Press, Princeton, New Jersey, USA

DENA, 2011, Dena Grid Study II: Integration of Renewable Energy Sources in the German Power Supply System from 2015 - 2020 with an Outlook to 2025, Deutsche Energie-Agentur GmbH (dena)

DOE, 2006, Benefits of demand response in electricity markets and recommendations for achieving them. A report to the United States Congress Pursuant to Section 1252 of the Energy Policy Act of 2005, http://energy.gov/oe/downloads/benefits-demand-response-electricity-markets-andrecommendations-achieving-them-report, accessed 02.02.2014

EPEX Spot, 2013, European Power Exchange, <www.epexspot.com> accessed on 11/11/2013 
Erdirik-Dogan, M, and Grossmann, I. E., 2008, Simultaneous planning and scheduling of single-stage multi-product continuous plants with parallel lines, Computers and Chemical Engineering, 32, 26642683

Floudas, C., and Lin, X., 2004, Continuous-time versus discrete-time approaches for scheduling of chemical processes: a review, Computers \& Chemical Engineering, 28, 11, 2109-2129

Guignard, M., Kim, S., 1987, Lagrangean decomposition: A model yielding stronger Lagrangean bounds , Mathematical Programming, 39, 215-228

Hadera, H. and Harjunkoski, I., 2013, Continuous-time Batch Scheduling Approach for Optimizing Electricity Consumption Cost, Computer Aided Chemical Engineering, Elsevier, 32, 403-408

Hadera, H., Harjunkoski, I., Grossmann, I. E., Sand, G., Engell, S., 2014, Steel production scheduling under time-sensitive electricity cost, Computer Aided Chemical Engineering, Elsevier, 33, 373-378

Hait, A. and Artigues, C., 2011a, On electrical load tracking scheduling for a steel plant, Computers \& Chemical Engineering, 35, 12, 14, 3044-3047

Hait, A. and Artigues, C., 2011b, A hybrid CP/MILP method for scheduling with energy costs, European Journal of Industrial Engineering, 5, 4, 471-489

Harjunkoski, I. and Grossmann, I. E., 2001, A decomposition approach for the scheduling of a steel plant production, Computers \& Chemical Engineering, 25, 11-12, 1647-1660

Harjunkoski, I. and Sand, G., 2008, Flexible and configurable MILP models for meltshop scheduling optimization, Computer Aided Chemical Engineering, 25, 677-682

Harjunkoski, I., Maravelias, C., Bongers, P., Castro, P., Engell, S., Grossmann, I. E., Hooker, J., Méndez, C., Sand, G., Wassick, J., 2014, Scope for industrial applications of production scheduling models and solution methods, Computers \& Chemical Engineering, 62, 161-193

Ierapetritou, M. G., Wu, D., Vin, J., Sweeny, P., Chigirinskiy, M., 2002, Cost Minimization in an Energy-Intensive Plant Using Mathematical Programming Approaches, Industrial and Engineering Chemistry Research, 41, 5262

Iyer, R. R. and Grossmann, I. E., 1998, A Bilevel Decomposition Algorithm for Long-Range Planning of Process Networks, Industrial \& Engineering Chemistry Research, 37, 2, 474-481

Li, J., Xiao, X., Tang, O., Floudas, C. A., 2012, Production Scheduling of a Large-Scale Steelmaking Continuous Casting Process via Unit-Specific Event-Based Continuous-Time Models: Short-Term and Medium-Term Scheduling, Industrial \& Engineering Chemistry Research, 51, 21, 7300-7319

Li, P., Wendt, M., Wozny, G., 2003, Optimal production planning under uncertain market conditions, In: Bingzhen Chen and Arthur W. Westerberg, Editor(s), Computer Aided Chemical Engineering, Elsevier, 15, 511-516

Maravelias, C., 2012, General framework and modeling approach classification for chemical production scheduling, AIChE Journal, 58, 6, 1812-1828

Méndez, C. A., Cerdá, J., Grossmann, I. E., Harjunkoski, I., Fahl, M., 2006, State-of-the-art review of optimization methods for short-term scheduling of batch processes, Computers \& Chemical Engineering, 30, 6-7, 913-946 
Mitra, S., Grossmann, I. E., Pinto, J. M., Arora, N., 2012, Optimal production planning under timesensitive electricity prices for continuous power-intensive processes, Comp. and Chem. Eng., 38, 171184

Mitra, S, Sun, L., Grossmann, I. E., 2013, Optimal Scheduling of Industrial Combined Heat and Power Plant under Time-sensitive Electricity Prices, Energy 54, 194-211

NERC, 2007, Data Collection for Demand-Side Management for Quantifying its Influence on Reliability, http://www.nerc.com/docs/pc/drdtf/NERC_DSMTF_Report_040308.pdf, accessed 02.02.2014

Nolde, K. and Morari, M., 2010, Electrical load tracking scheduling of a steel plant, Comp. and Chem. Eng, 34,11, 1899-1903

Paulus, M., Borggrefe, F., 2011, The potential of demand-side management in energy-intensive industries for electricity markets in Germany, Applied Energy, 88, 2, 432-441

Tang, L., Liu, J., Rong, A., Yang, Z., 2001, A review of planning and scheduling systems and methods for integrated steel production, European Journal of Operational Research, 133, 1, 1-20

Todd, D., Caufield, M., Helms, B., Starke, M., Kirby, B., Kueck, J., 2009, Providing Reliability Services through Demand Response: A Preliminary Evaluation of the Demand Response Capabilities of Alcoa Inc. Project report to US Department of Energy contract DE-AC05-00OR22725

Vujanic, R., Mariéthoz, S., Goulart, P. J., Morari, M., 2012, Robust Integer Optimization and Scheduling Problems for Large Electricity Consumers, American Control Conference, Montreal, Canada, 3108-3113

Wu, D., Ierapetritou, M. G., 2003, Decomposition approaches for the efficient solution of short-term scheduling problems, Computers and Chemical Engineering, 27, 8, 1261-1276

Xu, C., Sand, G., Harjunkoski, I., Engell, S., 2012, A new heuristic for plant-wide schedule coordination problems: The intersection coordination heuristic, Computers \& Chemical Engineering, 42, $152-167$

Zhang, Y. and Tang, L., 2010, Production Scheduling with Power Price Coordination in Steel Industry, Power and Energy Engineering Conference (APPEEC), 28-31 March 2010, pp. 1-4 


\section{Appendix A}

The literature based extension of energy-awareness for continuous-time scheduling models uses six different cases of how a task can contribute to electricity consumption within a considered time slot:

1. A task is processed entirely within the time slot.

Processing within a time slot means that stage's finishing time $t_{p, s t}^{f}$ occurs before the time slot's finishing time $\tau_{s}$ and stage's starting time $t_{p, s t}^{s}$ occurs later than the time slot's starting time $\tau_{s-1}$. For this case the binary variable $A_{p, s, s t}$ will be true, thus equations using Big-M formulation are written as in Eq. A.1- A.2. The duration of processing within the slot will in this case be equal to the processing time of the task itself.

$$
\begin{array}{ll}
t_{p, s t}^{f} \leq \tau_{s}+\left(M-\tau_{s}\right)\left(1-A_{p, s, s t}\right) & \forall p \in P, s \in S, s t \in S T \text { (A.1) } \\
t_{p, s t}^{s} \geq \tau_{s-1}-\tau_{s-1}\left(1-A_{p, s, s t}\right) & \forall p \in P, s \in S, s t \in S T \text { (A.2) }
\end{array}
$$

2. A task starts before and finishes within the time slot.

Second case occurs if stage's start time $t_{p, s t}^{s}$ occurs before the lower boundary of the considered slot (Eq. A.5), however the stage's finish time $t_{p, s t}^{f}$ is placed within the slot (Eq. A.3- A.4). For this case the binary variable $B_{p, s, s t}$ will be true. Processing time contribution of the task within the slot is equal to the tasks' finishing time $t_{p, m}^{f}$ minus the lower boundary $\tau_{s-1}$ of the considered time slot.

$$
\begin{aligned}
& t_{p, s t}^{f} \geq \tau_{s-1}-\tau_{s-1}\left(1-B_{p, s, s t}\right) \quad \forall p \in P, s \in S, s t \in S T \text { (A.3) } \\
& t_{p, s t}^{f} \leq \tau_{s}+\left(M-\tau_{s}\right)\left(1-B_{p, s, s t}-A_{p, s, s t}\right) \quad \forall p \in P, s \in S, s t \in S T \text { (A.4) } \\
& t_{p, s t}^{s} \leq \tau_{s-1}+\left(M-\tau_{s-1}\right)\left(1-B_{p, s, s t}\right) \forall p \in P, s \in S, s t \in S T \text { (A.5) }
\end{aligned}
$$

3. A task starts within and finishes after the time slot.

Similarly to the second case, the task's start time $t_{p, s t}^{S}$ occurs within the considered time interval (Eq. A.7- A.8) and at the same time finishing time $t_{p, s t}^{f}$ is placed after the upper boundary of the slot (Eq. A.6). For this case the binary variable $C_{p, s, s t}$ will be true. The time a task spent within the slot will equal to the upper boundary $\tau_{s}$ of the slot minus the start time $t_{p, m}^{S}$ of the task.

$$
\begin{aligned}
& t_{p, s t}^{f} \geq \tau_{s}-\tau_{s}\left(1-C_{p, s, s t}\right) \quad \forall p \in P, s \in S, s t \in S T \text { (A.6) } \\
& t_{p, s t}^{s} \geq \tau_{s-1}-\tau_{s-1}\left(1-C_{p, s, s t}-A_{p, s, s t}\right) \quad \forall p \in P, s \in S, s t \in S T \text { (A.7) } \\
& t_{p, s t}^{s} \leq \tau_{s}+\left(M-\tau_{s}\right)\left(1-C_{p, s, s t}\right) \quad \forall p \in P, s \in S, s t \in S T \text { (A.8) }
\end{aligned}
$$

\section{A task over-spans the time slot.}

When duration of the task is longer than the time interval itself there might be a case when it overspans the interval. This occurs only when the start time of the task $t_{p, s t}^{s}$ is placed before the lower boundary of the time slot (Eq. A.10) and at the same time the finish time $t_{p, s t}^{f}$ of task occurs after the 
upper bound of the slot (Eq. A.9). For this case the binary variable $D_{p, s, s t}$ will be true. Then, the amount of time the task contributed to the time slot will be equal to the length of the time slot itself $\left(\tau_{s}-\tau_{s-1}\right)$.

$t_{p, s t}^{f} \geq \tau_{s}-\tau_{s}\left(1-D_{p, s, s t}-C_{p, s, s t}\right) \quad \forall p \in P, s \in S, s t \in S T$ (A.9)

$t_{p, s t}^{s} \leq \tau_{s-1}+\left(M-\tau_{s-1}\right)\left(1-D_{p, s, s t}-B_{p, s, s t}\right) \quad \forall p \in P, s \in S, s t \in S T$ (A.10)

5. A task starts and finishes before the considered time slot.

Here both the starting time $t_{p, s t}^{s}$ and finishing time $t_{p, s t}^{f}$ takes place before the starting of the considered time interval $\tau_{s-1}$. For this case the binary variable $E_{p, s, s t}$ will be true when finishing time $t_{p, s t}^{f}$ occurs before the considered time slot, as in Eq. (A.11).

$t_{p, s t}^{f} \leq \tau_{s-1}+\left(M-\tau_{s-1}\right)\left(1-E_{p, s, s t}\right) \forall p \in P, s \in S, s t \in S T$

6. A task starts and finishes after the considered time slot.

Here both the starting time $t_{p, s t}^{s}$ and finishing time $t_{p, s t}^{f}$ takes place after the finishing of the considered time interval $\tau_{s}$. For this case the binary variable $F_{p, s, s t}$ will be true when starting time $t_{p, s t}^{s}$ occurs later than upper bound of the considered time slot, as in Eq. (A.12).

$t_{p, s t}^{s} \geq \tau_{s}-\tau_{s}\left(1-F_{p, s, s t}\right) \quad \forall p \in P, s \in S, s t \in S T$ (A.12)

The big-M value is set to be the end of the scheduling horizon. The formulation is improved compared to Nolde and Morari (2010) by introducing second binary in the Big-M equations of similar boundary conditions as in Eq. A.4, A.7, A.9, A.10. To complete the formulation, an important constraint ensuring that there is only one of the six binaries true for a task has to be enforced, as in Eq. (A.13).

$A_{p, s, s t}+B_{p, s, s t}+C_{p, s, s t}+D_{p, s, s t}+E_{p, s, s t}+F_{p, s, s t}=1 \quad \forall p \in P, s \in S, s t \in S T$

With the help of the binaries being true for respective cases of task-time slot relation, it is possible to capture the amount of time a given task was processed in a particular time slot. The task's consumption within the slot can be accounted for by multiplying time spent with a parameter of specific electricity consumption of the task. Therefore, with summation of all tasks the total electricity consumption in the time slot is captured with the Equation A.14. The equation is divided by 60 to convert the unit from $M W \min$ into $M W h$. In the equation two problems arise. First, there are two nonlinearities from the product of binary and continuous variable. Second, the equation do not account for the fact that one of the machines in the stage does not process a task.

$q_{s}=\sum_{p, s t, m \in S M_{s t, m}} h_{p, m}\left(A_{p, s, s t} \cdot \tau_{p, m}+B_{p, s, s t}\left(t_{p, s t}^{f}-\tau_{s-1}\right)+C_{p, s, s t}\left(\tau_{s}-t_{p, s t}^{s}\right)+D_{p, s, s t}\left(\tau_{s}-\right.\right.$ $\left.\left.\tau_{s-1}\right)\right) \frac{1}{60} \quad \forall s \in S(\mathrm{~A} .14)$

In order to deal with the latter problem, a set of auxiliary variables can be designed for which those tasks not processing a product will have the time contribution to the slot put to zero. That means, whenever a product is not assigned to a machine the binaries of respective six cases shall be put to zero. For the first case with $A_{p, s, s t}$ binary, it can only be true when assignment binary $X_{m, p}$ is true, as in Eq. A.15- A.16. Similarly for the $D_{p, s, s t}$ binary as in Equation A.17- A.18.

$a_{p, m, s t, s} \geq A_{p, s, s t}-\left(1-X_{m, p}\right) \quad \forall p \in P, m \in M, s t \in S T, s \in S,\{s t, m\} \in S M$ 


$$
\begin{aligned}
& \left.a_{p, m, s t, s} \leq A_{p, s, s t}+1-X_{m, p}\right) \quad \forall p \in P, m \in M, s t \in S T, s \in S,\{s t, m\} \in S M \quad \text { (A.16) } \\
& d_{p, m, s t, s} \geq A_{p, s, s t}-\left(1-X_{m, p}\right) \quad \forall p \in P, m \in M, s t \in S T, s \in S,\{s t, m\} \in S M \\
& d_{p, m, s t, s} \leq A_{p, s, s t}+1-X_{m, p} \quad \forall p \in P, m \in M, s t \in S T, s \in S,\{s t, m\} \in S M \quad \text { (A.18) }
\end{aligned}
$$

For the other cases of $B_{p, s, s t}$ and $C_{p, s, s t}$ by designing the auxiliary variable we also can deal with the nonlinearities, by applying an exact linearization method. The auxiliary variables will have the value of the time contribution of the respective binary case only both the case binary is true and the assignment is true as well. The constraints for the two cases are shown in Equations A.19- A.26.

$b_{p, m, s t, s} \geq t_{p, s t}^{f}-\tau_{s-1}-\left(M-\tau_{s-1}\right)\left(2-B_{p, s, s t}-X_{m, p}\right) \quad \forall p \in P, m \in M, s t \in S T, s \in$ $S,\{s t, m\} \in S M$

$b_{p, m, s t, s} \leq t_{p, s t}^{f}-\tau_{s-1}+\tau_{s-1}\left(2-B_{p, s, s t}-X_{m, p}\right) \quad \forall p \in P, m \in M, s t \in S T, s \in S,\{s t, m\} \in S M$ (A.20)

$b_{p, m, s t, s} \leq\left(\tau_{s}-\tau_{s-1}\right)\left(1-B_{p, s, s t}+X_{p, m}\right) \quad \forall p \in P, m \in M, s t \in S T, s \in S,\{s t, m\} \in S M$

$b_{p, m, s t, s} \leq\left(\tau_{s}-\tau_{s-1}\right) \cdot B_{p, s, s t} \forall p \in P, m \in M, s t \in S T, s \in S,\{s t, m\} \in S M \quad$ (A.22)

$c_{p, m, s t, s} \geq \tau_{s}-t_{p, s t}^{s}-\tau_{s}\left(2-C_{p, s, s t}-X_{m, p}\right) \quad \forall p \in P, m \in M, s t \in S T, s \in S,\{s t, m\} \in S M$

$c_{p, m, s t, s} \leq \tau_{s}-t_{p, s t}^{s}+\left(M-\tau_{s}\right)\left(2-C_{p, s, s t}-X_{m, p}\right) \quad \forall p \in P, m \in M, s t \in S T, s \in S,\{s t, m\} \in S M$ (A.24)

$c_{p, m, s t, s} \leq\left(\tau_{s}-\tau_{s-1}\right)\left(1-C_{p, s, s t}+X_{m, p}\right) \quad \forall p \in P, m \in M, s t \in S T, s \in S,\{s t, m\} \in S M$

$c_{p, m, s t, s} \leq\left(\tau_{s}-\tau_{s-1}\right) \cdot C_{p, s, s t} \quad \forall p \in P, m \in M, s t \in S T, s \in S,\{s t, m\} \in S M \quad$ (A.26)

With the help of the auxiliary variables the final constraint for electricity consumption accounting can be changed from Equation A.14 to the one shown in Equation A.27.

$q_{s}=\sum_{p \in P, s t \in S T, m \in S M_{s t, m}} h_{p, m}\left(a_{p, m, s t, s} \tau_{p, m}+b_{p, m, s t, s}+c_{p, m, s t, s}+d_{p, m, s t, s}\left(\tau_{s}-\tau_{s-1}\right)\right) \frac{1}{60} \quad \forall s \in$ $S \quad$ (A.27) 


\section{Appendix B}

Table B.1. Calculation of bounds for task start time

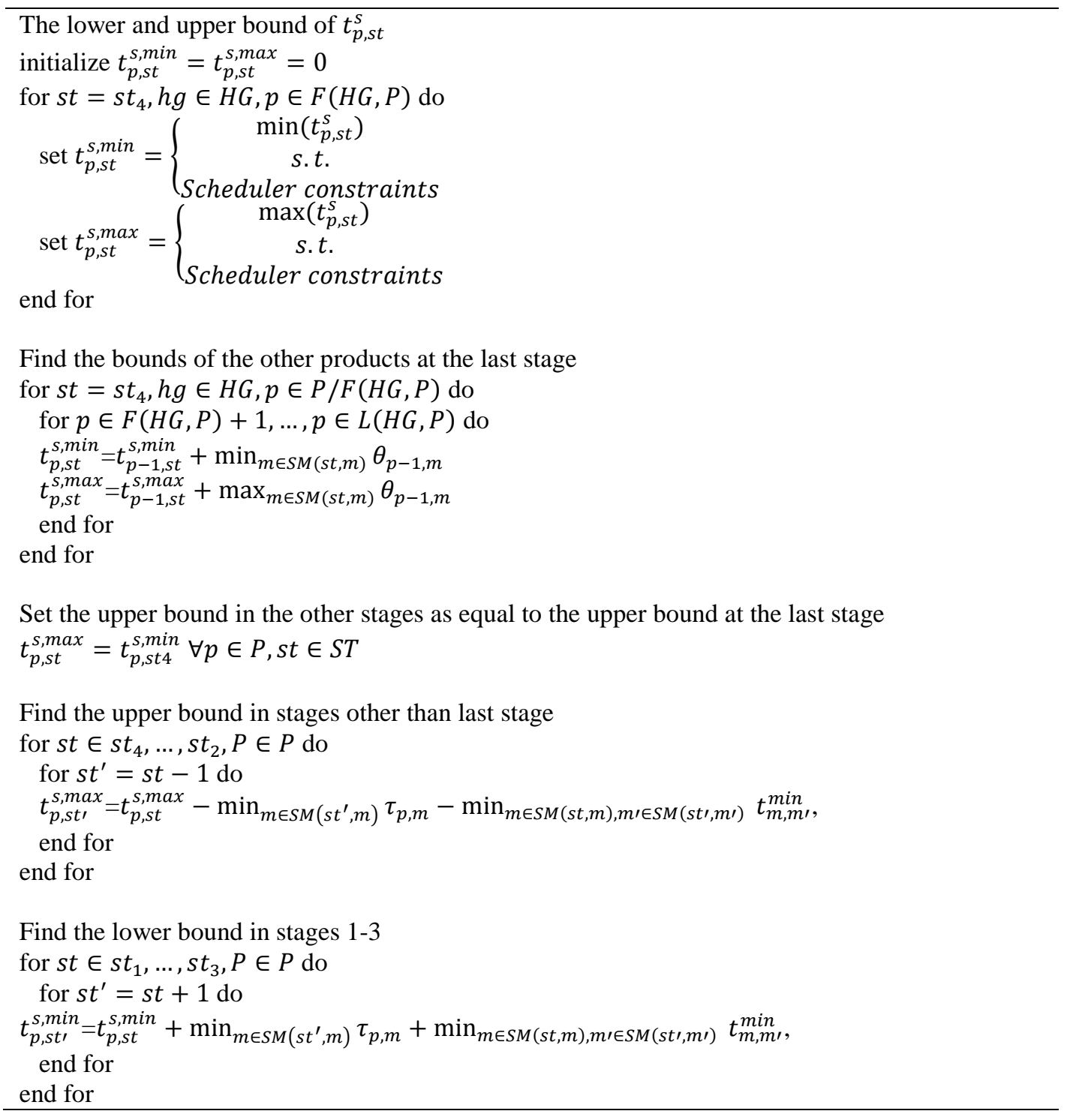

